九州大学学術情報リポジトリ

Kyushu University Institutional Repository

\title{
Approximate weight functions of stress intensity factor for a wide range shapes of surface and an embedded elliptical crack
}

Takaki, Yoshifumi

Doctoral' Course Student, Department of Civil and Structural Engineering, Graduate School of Engineering, Kyushu University

Gotoh, Koj i

Department of Marine Systems Engineering, Kyushu University

http://hdl. handle. net/2324/4751320

出版情報: Marine structures. 70 (102696)，2020-03. International Ship and 0ffshore Structures Congress

バージョン：

権利関係 : 


\title{
Approximate weight functions of stress intensity factor for a wide range shapes of surface and an embedded elliptical crack
}

\author{
Yoshifumi Takaki \\ Doctoral Course Student, Department of Civil and Structural Engineering, Graduate \\ School of Engineering, Kyushu University
}

Koji Gotoh*

Professor, Department of Marine Systems Engineering, Faculty of Engineering, Kyushu University,

744 Motooka, Nishi-ku, Fukuoka, 819-0395, Japan

$+81-92-802-3247$

$+81-92-802-3368$

gotoh@nams.kyushu-u.ac.jp

* Corresponding author

\begin{abstract}
This paper describes the application of weight function for stress intensity factor in a surface crack with a wide range of crack shapes. Based on the weight function representation proposed by Wang and Glinka (2009), an extended formulation of the weight function for surfaces and embedded cracks is performed. The unknown parameters in the formulations were determined from reference values of stress intensity factor for a surface and an embedded crack given by finite element analysis with the $\mathrm{J}$ integral method. The effectiveness of the proposed weight functions has been verified by comparing with the stress intensity factors given by finite element analysis and available approximated solutions under several loading conditions.
\end{abstract}

Keyword: Weight function; Stress intensity factor; surface crack; embedded crack 


\section{Nomenclature}

A

$a$

B

C

c

$d$

$\mathrm{F}_{0}(x, y)$

M

$m(X, Y ; x, y ; a, c)$

$m(x, y)$

m

n

$\mathrm{P}(x, y)$

Q

$\mathrm{Q}(x, y)$

$R(\varphi)$

$t$

$X, Y$

$x, y$

$\alpha$ vertex on minor axis of ellipse; the deepest point of a surface crack and the point on the crack front closest to the plate front surface of an embedded crack half-length of minor axis for an elliptical crack; half crack depth in case of an embedded crack, and crack depth in case of a surface crack vertex on minor axis of ellipse; the point farthest from the plate front surface of an embedded crack front vertex on major axis of ellipse; the surface point of a surface crack half-length of major axis for an elliptical crack; half crack width distance from plate surface to vertex on minor axis of ellipse point load location on crack face (see Fig. 1) coefficient to define the weight function point load weight function for the stress intensity factor at $(x, y)$ in the case that the aspect ratio of a crack is $a / c$ at loading point $(X, Y)$

abbreviation for $m(X, Y ; x, y ; a, c)$

exponent to describe the stress distribution on crack face

exponent to describe the stress distribution on crack face

point along the crack front under consideration (see Fig. 1)

complete elliptical integral of second kind

intersection point of the extension of the line $\mathrm{OF}_{0}$ and the front edge of the ellipse (see Fig. 1)

radius of polar coordinate for points along crack front edge (see Fig.1)

Plate thickness

Cartesian coordinates to define the point

reference point on the crack front edge

aspect ratio of ellipse, $\alpha=a / c$ 
dimensionless crack depth, $\zeta=a / t$

dimensionless distance form plate surface to vertex on minor axis of ellipse, $\eta=d / a$

$\theta$

angle of polar coordinates for point P (see Fig. 1)

$\xi$

shortest distance from the load point $\mathrm{F}_{0}$ to the crack front edge $\mathrm{S}$

$\rho$

distance between the load point $\mathrm{F}_{0}$ and the point $\mathrm{P}$ along crack front under

$\sigma(x, y)$

consideration

$\sigma_{0}$

stress on the crack face in case of no crack state

unit stress

$\varphi$

angle of polar coordinates for load point $\mathrm{F}_{0}$ (see Fig. 1) 


\section{Introduction}

Most fatigue surface cracks in welded structures initiate and grow under repeated coalescence. These surface cracks finally merge to a single surface crack. Subsequently, this surface crack grows and penetrates the plate. The shape of fatigue cracks during most stages of the total fatigue life is surface cracks. The stress intensity factor is widely applied to evaluate the strength or fatigue crack growth history of cracked bodies. Hence, it is necessary to calculate the stress intensity factor to evaluate the structural integrity of cracked bodies. Though many expressions of the stress intensity factor for a surface crack have been proposed, most of them can be applied only under a simple stress distribution, for example, uniform tension and pure bending. Approximate expression of stress intensity factor for a surface crack under remote uniform or linear stress distribution was proposed by Newman and Raju [1, 2]. Although this expression is widely accepted, it is impossible to apply it under arbitrary loading conditions, which sometimes cause complex stress distributions over supposed crack surfaces.

The weight function method has been widely applied to calculate stress intensity factors for its distinctive advantage of separating the loading and the geometry. Once the weight function is identified for a given cracked geometry, the stress intensity factor under arbitrary loading conditions applied to the same cracked body can be calculated by using the same weight function. Because the concept of weight function was originally introduced $[3,4]$ for one-dimensional through-thickness cracks, most studies reported in the literature have concentrated on one-dimensional cracks. The methods of obtaining the weight functions for onedimensional cracks have been well developed; see [5-7] for example. Representative weight functions have even been published as a handbook [8].

However, a method to identify the weight functions for surface and embedded elliptical cracks, which are widely found in welded joints and other engineering components, is still not well developed. For twodimensional cracks, close-form exact weight functions are available only for very limited cases: the circular crack and the half-plane crack in an infinite body [9].

Useful approximate weight functions for an embedded elliptical shape crack were proposed by Wang and Glinka [10]. One of authors [11] has improved the weight functions of Wang and Glinka [10] to be 
applicable to an embedded crack and a surface crack in a wider range of shapes. The applicable crack shape for the improved weight function is for the case where the aspect ratio of an elliptical crack is less than 1.0. However, it might be necessary to calculate the stress intensity factor for a wider range of crack shapes. When fatigue cracks propagate in thick steel plates used in large container ships, the aspect ratio of the crack shape may increase, that is, exceed 1.0. Such a phenomenon can also occur in the case of multi-axis loads.

Therefore, this study developed a weight function applicable to surface cracks and embedded cracks with various shapes based on the expression of Wang and Glinka [10].

\section{Theoretical background and improvements offered by the weight function}

In general, the shape of a two-dimensional crack is approximated as semi-elliptical for a surface crack and elliptical for an embedded crack. The coordinate system to define the point loading position, crack shape, and reference points on the crack front edge is shown in Fig. 1. The center of the ellipse is the origin. The $X$ axis and $Y$-axis are set up in the direction of the major and minor axes, respectively. The $Y$-axis direction is the plate thickness direction. The $X$-axis direction is the direction of the surface crack width and is orthogonal to the $Y$-axis. For a surface crack, the $X$-axis is set on the front surface of the plate, and the aspect ratio $a / c$ is applied to express the crack shape.

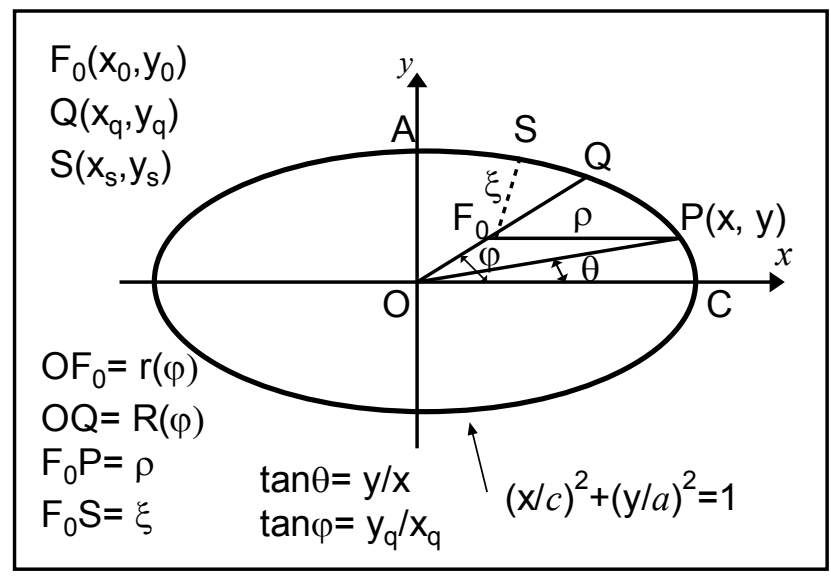

Fig. 1 Coordinate system for defining the weight function 
In the expression of Wang and Glinka [10], the stress intensity factor $K$ of a surface and an embedded crack applying a stress distribution $\sigma(x, y)$ over crack faces can be described by using the weight function, $m(X$, $Y ; x, y ; a, c)$, and the general expression of $K$ is described by Eq. (1).

$$
K(x, y)=\int_{-a}^{a} \int_{-c}^{c} \sigma(X, Y) m(X, Y ; x, y ; a, c) d X d Y
$$

Hereafter, the weight function $m(X, Y ; x, y ; a, c)$ is abbreviated as $m(x, y)$. The general expression of weight function, $m(x, y)$, in Eq. (1) is described as follows [10]:

$$
m(x, y)=\frac{\sqrt{2 \xi}}{\pi^{3 / 2} \rho^{2}}[1+M\{1-r(\varphi) / R(\varphi)\}]
$$

Although the coefficient in Eq. (2), $M$, is expressed as a power series function of $\theta$ and $\alpha$ [9], the following problems were confirmed by one of authors [11]:

(1) The application of the weight function for the stress intensity factor is not verified under more complex stress distribution given by polynomials of fourth order or higher.

(2) The accuracy of the stress intensity factor is poor depending on the crack shape, even under a stress distribution approximated by a polynomial with lower order than a cubic function. Figure 2 compares the stress intensity factor by weight functions $[10,12]$ under remote uniform stress conditions. Parameter $Q$ in the vertical axis of Fig. 2 is the complete elliptical integral of the second kind, approximated by the following empirical equation:

$$
Q=\left\{\begin{array}{cc}
1+1.464(a / c)^{1.65} & (a / c \leq 1.0) \\
{\left[1+1.464(c / a)^{1.65}\right](a / c)^{2}} & (a / c>1.0)
\end{array}\right.
$$

The white circles in Fig. 2 show the dimensionless stress intensity factors derived from the weight function of Wang and Glinka [10], and the black circles show the factors derived by the influence function method of Shiratori et al. [12]. It is confirmed from Fig. 2 that the behavior of the stress intensity factor obtained 
by the weight function [10] becomes unnatural as the value of $a / t$ increases.

(3) The validity of estimating the stress intensity factor of a surface and an embedded crack when the crack surface stress is distributed in the crack width direction has not been verified yet. The influence function proposed by Nagai et al. [13] can be applied when the aspect ratio is greater than 1.0. The aspect ratio verified by the influence function was only five case, 1.0, 2.0, 3.0, 4.0 and 8.0. Moreover, stress intensity factor between $a / c=4.0$ and $a / c=8.0$ was not verified

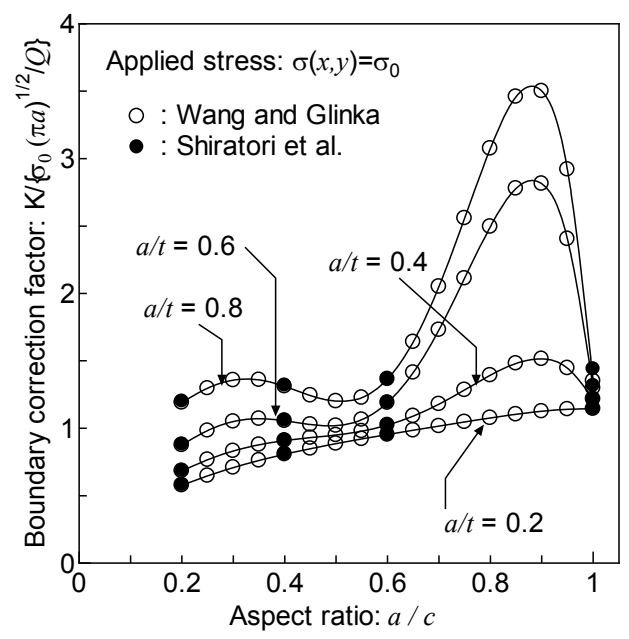

Fig. 2 Preliminary investigation of the validity of weight function method proposed by Wang and Glinka [10] (point C in Fig. 1)

Because the weight function represented by Eq. (2) has a reasonable theoretical background, the utility of Eq. (2) can be obtained by updating the coefficient $M$ so as to be applicable to a wider range of conditions. Moreover, the value of the stress intensity factor of the elliptical crack at the top of the minor and major axes corresponds to the value of the deepest and shallowest (surface) points of the crack. It is important to calculate the stress intensity factor at these points $(\theta=\pi / 2$ at the top of the minor axis, and $\theta=0$ at the top of the major axis) for the evaluation of the structural integrity of a component containing two-dimensional cracks.

In this study, the coefficient $M$ at these points was updated for a wider range of aspect ratios. The reference solutions of stress intensity factor were as follows:

(1) The influence function method of Shiratori et al. [12] and the empirical formula of Newman and Raju [1, 
2], which are well-known for a surface crack and an embedded crack, respectively. In the former method, the influence factor is defined as the stress intensity factor because of the unit load was applied to the finite element nodes. The stress intensity factor under the stress distribution that loads the crack can be calculated by superimposing the applied stress and the corresponding influence factor.

(2) Finite element analysis with the J-integral method for cases where the above mentioned methods cannot be applied due to a restriction on the crack shape. For example, there is no reference solution of the stress intensity factor under the stress distribution which changes in the direction of the crack width in the case of the surface crack. Commercial finite element (FE) software, including MSC Marc [14] and Abaqus [15], was applied. The calculation of the $J$ integral employed a path integration method (the number of paths was eight, and the average value of each $\mathrm{J}$ integral was adopted for the calculation of stress intensity factor) which was usually implemented by the same code. The FE model was built using hexahedral 20-node elements. The number of elements and number of nodes were different from model to model, but in all analysis models, the minimum dimension of elements along the crack front was about $0.05 \mathrm{~mm}$. Crack front length is $0.4 \mathrm{~mm}$. Symmetry plane of the plate is applied to a symmetric condition and bottom plane of the plate is applied to a support condition in finite element's analyzing. These boundary conditions of FE analysis adopted in this study are standard methods used to calculate SIF in FE analysis.

The weight function coefficient $M$ was inversely identified as a numerical value to correlate the values of stress intensity factor obtained by Eqs. (1) and (2) with the reference values. The results described as polynomial functions, such as the aspect ratio $\alpha$ and coefficients of each term, were identified by applying the solver function of Microsoft Excel [16]. The polynomial approximation results of $M$ are shown in the appendix.

\section{Weight function for a surface crack in finite plate depth}

The weight function of stress intensity factors for a surface crack was verified and updated. Figure 3 shows a plate with finite thickness containing a semi-elliptical surface crack to be evaluated. Coordinate systems 
are also shown in Fig. 3. Parameters to describe the crack tip locations are defined in Fig. 1.

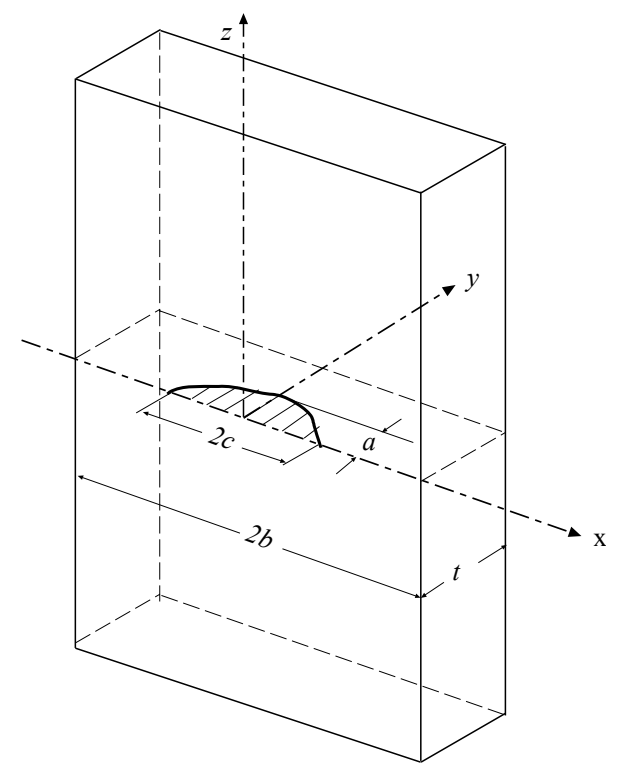

Fig. 3 Geometry and coordinate system of a semi-elliptical surface crack in a plate with finite thickness.

The applied stress distributions on the crack face are expressed by Eqs. (4) and (5).

$$
\begin{gathered}
\sigma(x, y)=\sigma_{0}(1-y / a)^{n},(n=0,1, \cdots, 6) \\
\sigma(x, y)=\sigma_{0}(1-x / c)^{n},(n=0,1, \cdots, 6)
\end{gathered}
$$

\subsection{Aspect ratio less than 1.0.}

A verification was performed for crack shapes with aspect ratios $a / c$ of $0.25,0.5,0.75$, and 1.0, dimensionless crack depths $a / t$ of $0.3,0.5$, and 0.8 and dimensionless crack width $c / b$ of 0.5 . Figure 4 compares the stress intensity factors under the stress field determined by Eq. (4) for the deepest point of surface crack (point A in Fig. 1) with the field for the surface of the crack (point $\mathrm{C}$ in Fig. 1). Figure 5 compares the stress intensity factors under the stress field of Eq. (5). The comparisons in the case that $a / t=0.5$ and $a / t=0.8$ are shown 
in Figs. 6 to 9. The relative error between stress intensity factors calculated with the weight function proposed in this study and reference solutions increases as the crack shape becomes more planar and the crack depth increases. However, the relative error for the verification range is within $10 \%$.

From the perspective of fatigue strength evaluation, accuracy of the stress intensity factor in the range of $a / t<0.3$ is important because the fatigue life for a small crack is longer than that for a large crack during the total fatigue life. Even though these small cracks are under a steep stress gradient field, it is acceptable for the stress distribution on a surface crack to be regarded as constant or linear. To verify the utility of the proposed expression for a small crack, the value of stress intensity factor in the case that $a / t$ is equal to 0.02 is compared with factors obtained by the formula of Newman and Raju [1]. Table 1 is a comparison result of the dimensionless stress factor for several crack shapes. The relative error of the stress intensity factors calculated by the weight function proposed in this study and those calculated by the formula of Newman and Raju is less than $10 \%$ except for one case (Point $\mathrm{C}$ on Table 1 , where $a / c$ is 1.0 and applied stress is $\sigma_{0}$ ). It is concluded that the proposed weight function makes it possible to determine the stress intensity factor for surface cracks with an aspect ratio less than 1 when stress is applied to the crack surface in the width and thickness directions.

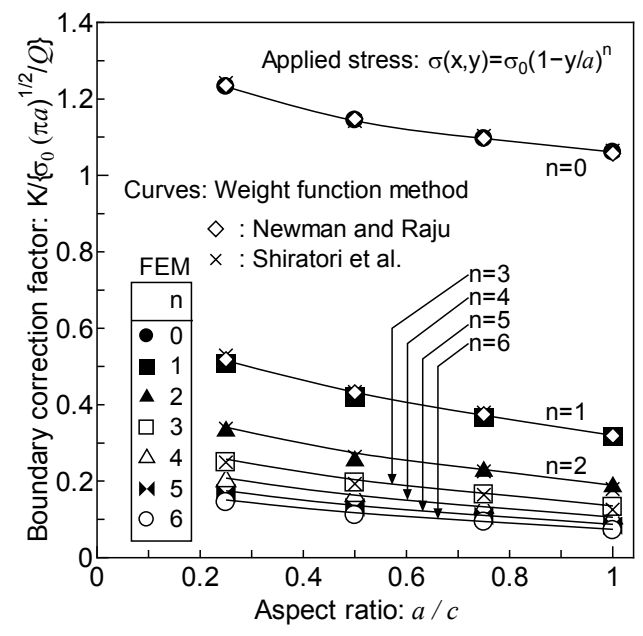

a) Point $\mathrm{A}(\varphi=\pi / 2)$

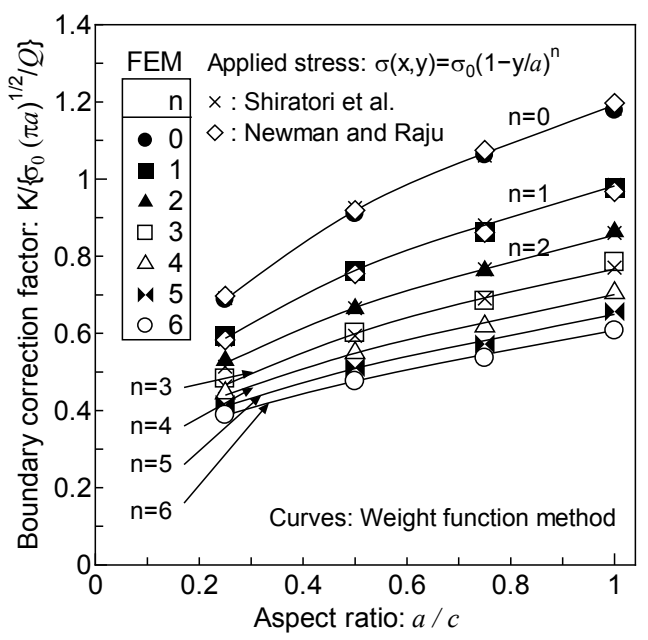

b) Point C ( $\varphi=0)$

Fig. 4 Dimensionless stress intensity factor under the applied stress distribution expressed by Eq. (4), $a / c \geq 1.0(a / t=0.3)$ 


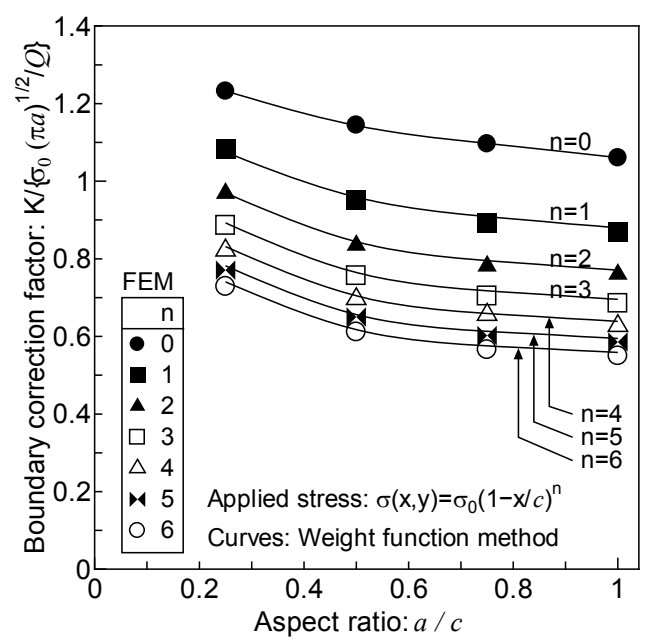

a) Point $\mathrm{A}(\varphi=\pi / 2)$

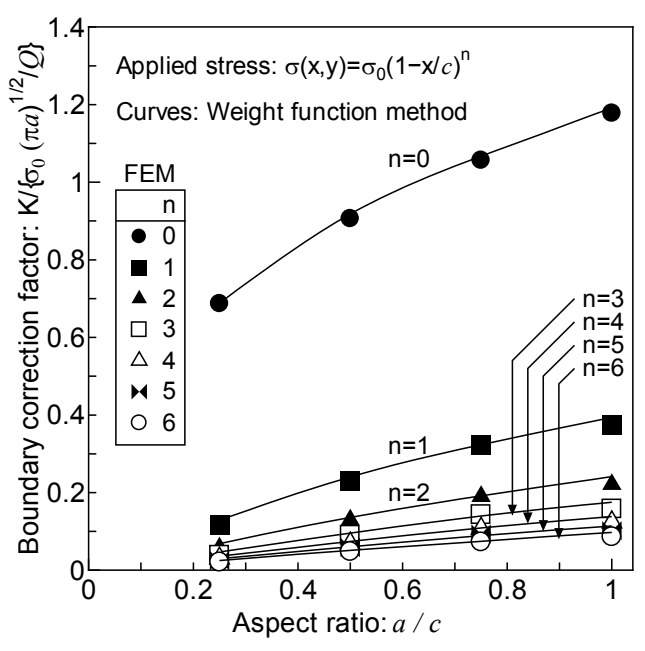

b) Point $\mathrm{C}(\varphi=0)$

Fig. 5 Dimensionless stress intensity factor under the applied stress distribution expressed by Eq. (5),

$$
a / c \leq 1.0(a / t=0.3)
$$

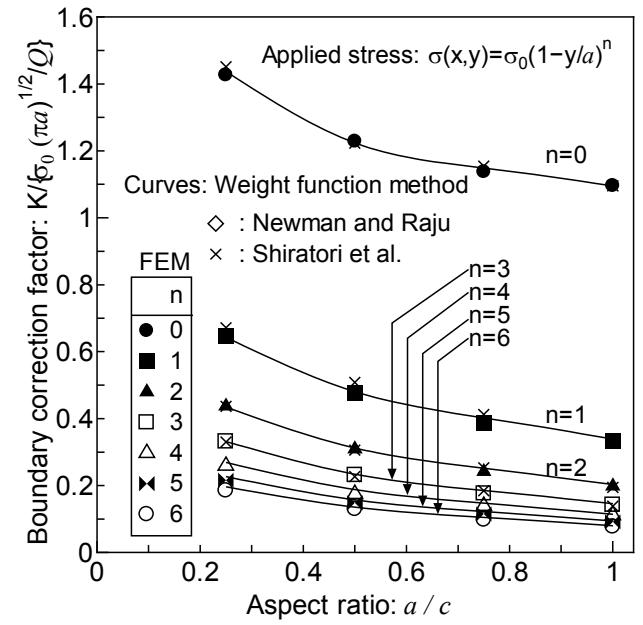

a) Point $\mathrm{A}(\varphi=\pi / 2)$

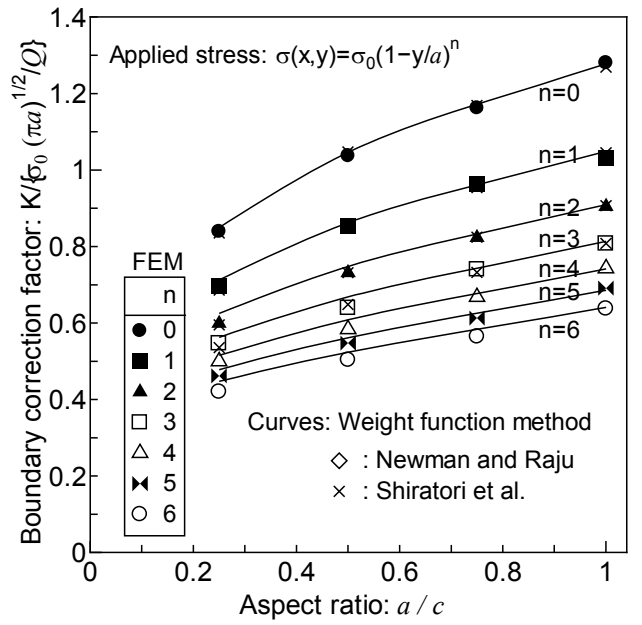

b) Point C $(\varphi=0)$

Fig. 6 Dimensionless stress intensity factor under the applied stress distribution expressed by Eq. (4),

$$
a / c \leq 1.0(a / t=0.5)
$$




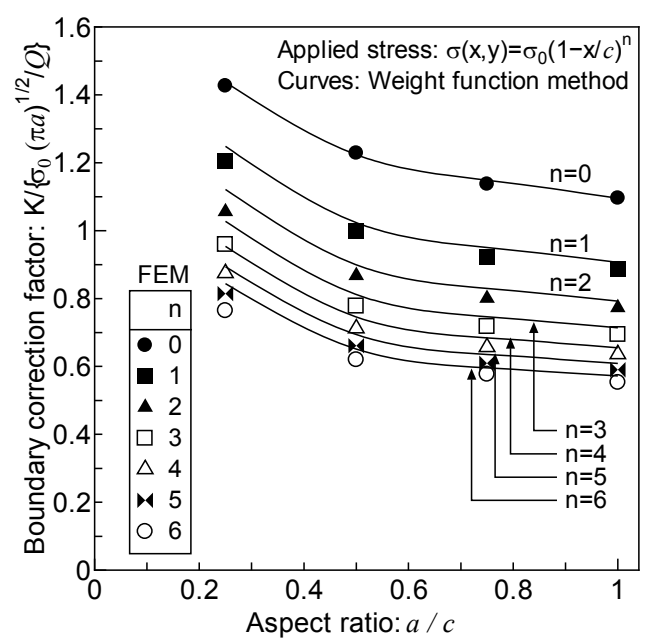

a) Point $\mathrm{A}(\varphi=\pi / 2)$

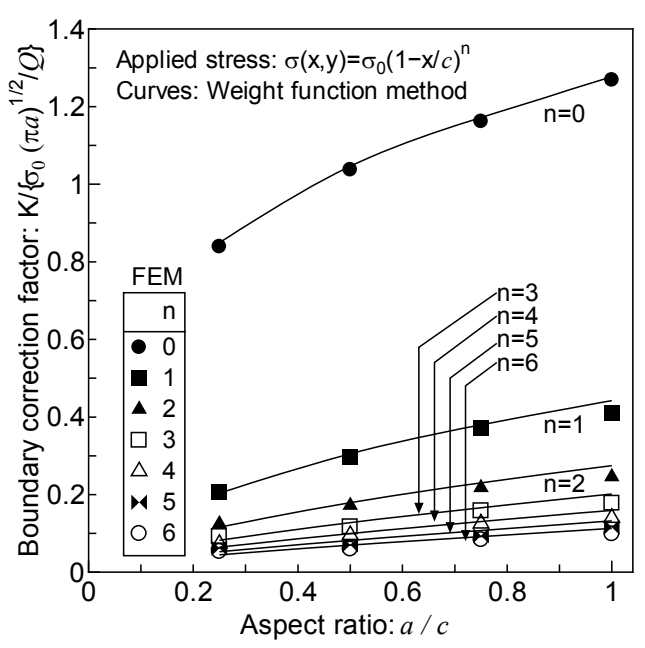

b) Point $\mathrm{C}(\varphi=0)$

Fig. 7 Dimensionless stress intensity factor under the applied stress distribution expressed by Eq. (5),

$$
a / c \leq 1.0(a / t=0.5)
$$

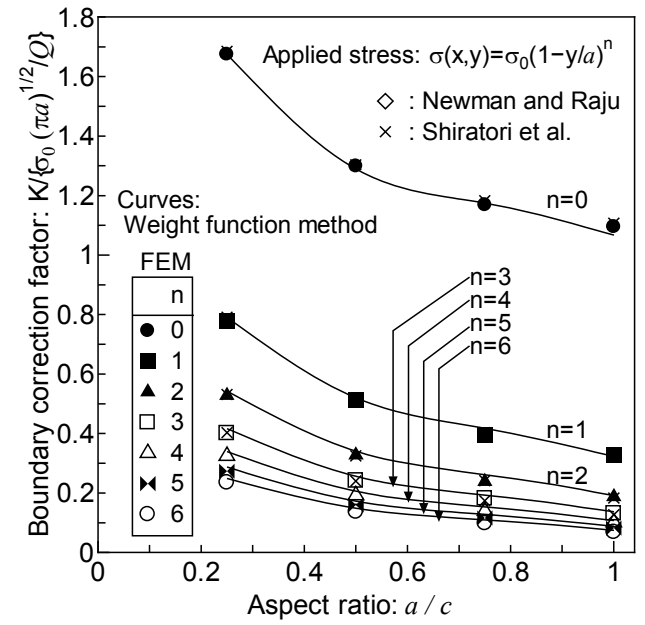

a) Point $\mathrm{A}(\varphi=\pi / 2)$

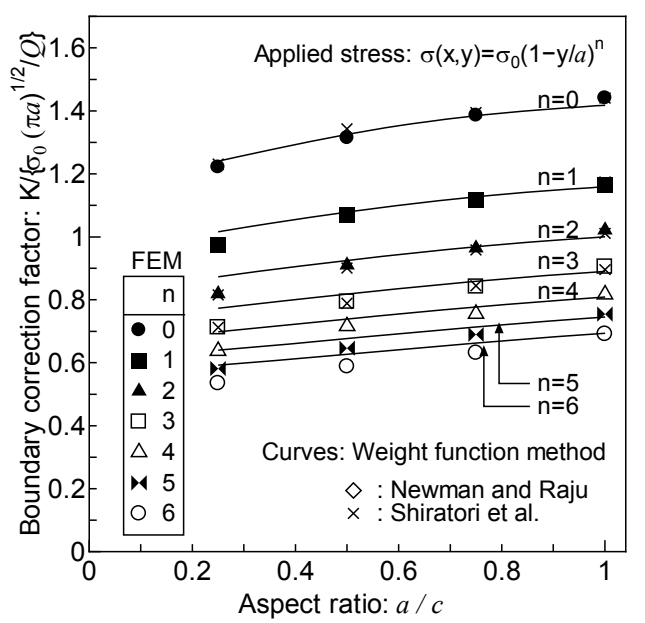

b) Point $\mathrm{C}(\varphi=0)$

Fig. 8 Dimensionless stress intensity factor under the applied stress distribution expressed by Eq. (4),

$$
a / c \leq 1.0 \quad(a / t=0.8)
$$




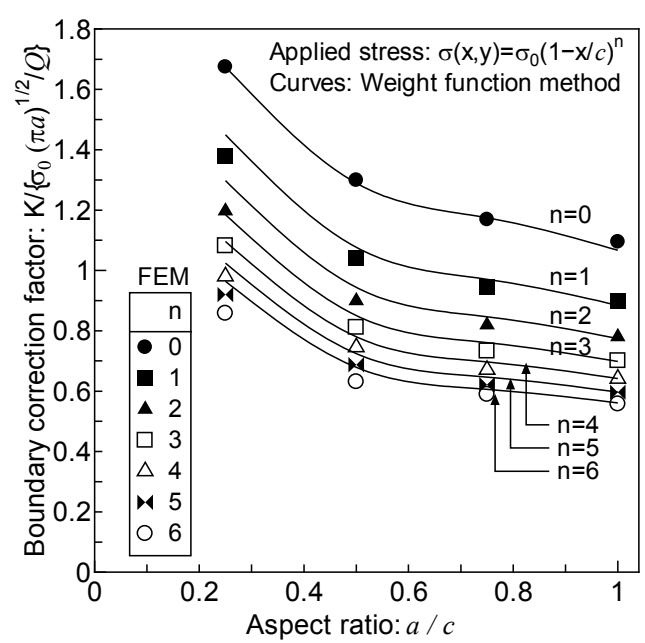

a) Point $\mathrm{A}(\varphi=\pi / 2)$

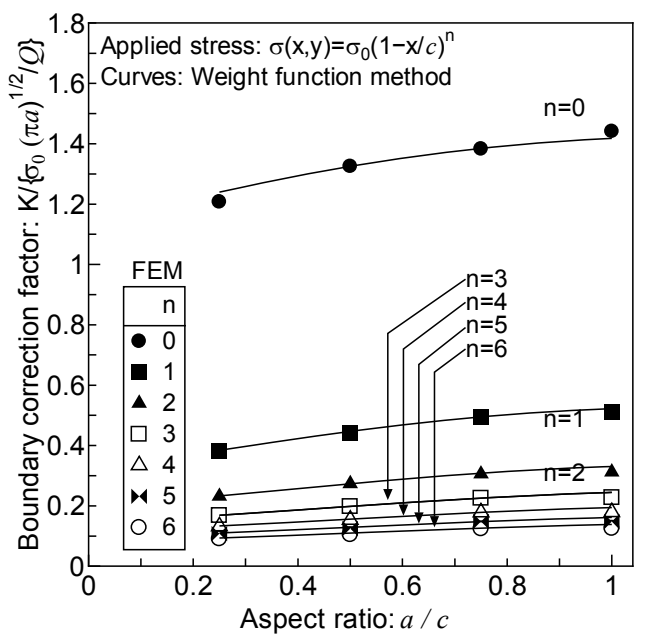

b) Point $\mathrm{C}(\varphi=0)$

Fig. 9 Dimensionless stress intensity factor under the applied stress distribution expressed by Eq. (5),

$$
a / c \leq 1.0 \quad(a / t=0.8)
$$

Table 1 Comparison of dimensionless stress intensity factor $(a / t=0.02)$

\begin{tabular}{|c|c|c|c|c|}
\hline \multicolumn{5}{|c|}{ Point A in Fig. $1(\varphi=\pi / 2)$. Applied stress: $\sigma(x, y)=\sigma_{0}$} \\
\hline$a / c$ & 0.25 & 0.5 & 0.75 & 1.0 \\
\hline DK $_{\mathrm{wf}}$ & 1.032 & 1.029 & 1.043 & 1.052 \\
\hline DK $_{\mathrm{NR}}$ & 1.109 & 1.086 & 1.063 & 1.041 \\
\hline Error (\%) & 6.911 & 5.219 & 1.898 & 1.096 \\
\hline Point C in Fig. 1 $(\varphi=0)$. Applied stress: $\sigma(x, y)=\sigma_{0}$ \\
\hline$a / c$ & 0.25 & 0.5 & 0.75 & 1.0 \\
\hline DK $_{\mathrm{wf}}$ & 0.599 & 0.817 & 0.916 & 1.000 \\
\hline DK $_{\mathrm{NR}}$ & 0.610 & 0.845 & 1.013 & 1.145 \\
\hline Error (\%) & 1.774 & 3.263 & 9.571 & 12.641 \\
\hline
\end{tabular}




\begin{tabular}{|c|c|c|c|c|}
\hline \multicolumn{5}{|c|}{ Point A in Fig. $1(\varphi=\pi / 2)$. Applied stress: $\sigma(x, y)=\sigma_{0}(1-y / a)$} \\
\hline$a / c$ & 0.25 & 0.5 & 0.75 & 1.0 \\
\hline DK $_{\mathrm{wf}}$ & 0.391 & 0.363 & 0.340 & 0.314 \\
\hline DK $_{\mathrm{NR}}$ & 0.421 & 0.392 & 0.367 & 0.343 \\
\hline Error (\%) & 7.234 & 7.335 & 7.288 & 8.434 \\
\hline Point C in Fig. 1 $(\varphi=0)$. Applied stress: $\sigma(x, y)=\sigma_{0}(1-y / a)$ \\
\hline$a / c$ & 0.25 & 0.5 & 0.75 & 1.0 \\
\hline DK $_{\mathrm{wf}}$ & 0.518 & 0.684 & 0.763 & 0.832 \\
\hline DK $_{\mathrm{NR}}$ & 0.501 & 0.678 & 0.799 & 0.887 \\
\hline Error (\%) & 3.293 & 0.948 & 4.457 & 6.179 \\
\hline
\end{tabular}

Note: $\mathrm{DK}_{\mathrm{wf}}=$ dimensionless stress intensity factor by the weight function method proposed in this paper; $\mathrm{DK}_{\mathrm{NR}}=$ dimensionless stress intensity factor determined by Newman and Raju's equation; and error = $\left(\left|\mathrm{DK}_{\mathrm{wf}}-\mathrm{DK}_{\mathrm{NR}}\right| / \mathrm{DK}_{\mathrm{NR}}\right) \times 100$.

\subsection{Aspect ratio from 1.0 to 10 .}

The weight function when the aspect ratio $a / c$ is from 1.0 to 10 was identified and verified for the dimensionless crack depths $a / t$ of $0.2,0.4,0.6$, and 0.8 . The cases of the verified crack shape (aspect ratio, $a / c$ ) in this paper is more than these in the paper by Nagai et al. [13]. The weight function coefficient $M$ was calculated inversely to correlate the values of stress intensity factor obtained by Eqs. (1) and (2) with the reference values. Considering the crack shape and importance of the evaluation of fracture initiation, the applied stress distribution of Eq. (4) is considered in this section. In these cases, the coefficient $M$ cannot be expressed by the polynomial function of $\theta$ and $a / c$. Parameter $n$, which gives the stress distribution, also influenced the weight function. Then, the coefficient $M$ is approximated by the polynomial function of $\theta$ and $a / c$ according to the value of $n$. These results are shown in the appendix. 
Comparison of stress intensity factors under the stress field of Eq. (4) for the deepest point of a surface crack (point $\mathrm{A}$ in Fig. 1) with those for the surface of the crack (point $\mathrm{C}$ in Fig. 1) is shown in Fig. 10. The comparisons for $a / t=0.4,0.6$, and 0.8 are shown in Figs. 11 to 13 . The relative error between stress intensity factors calculated with the weight function proposed in this study and reference solutions is within $10 \%$.

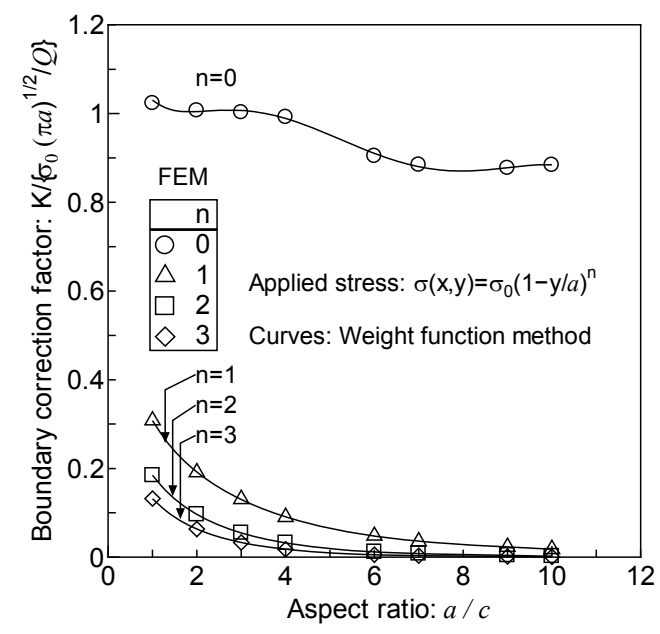

a) Point $\mathrm{A}(\varphi=\pi / 2)$

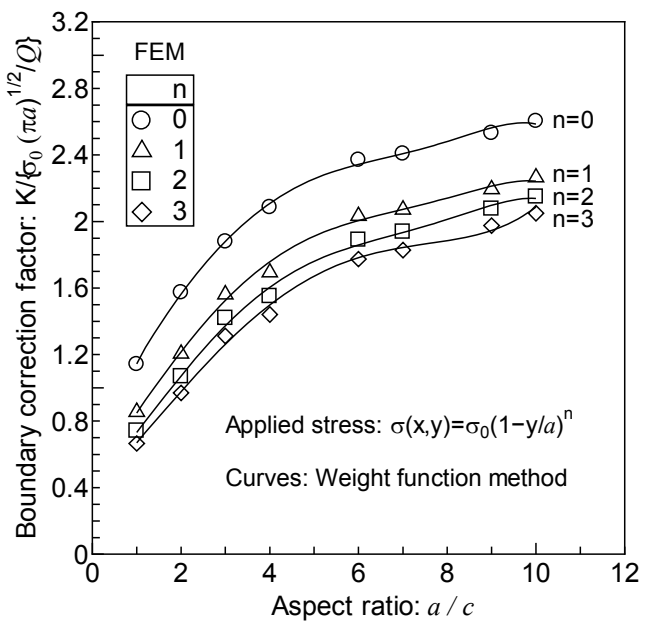

b) Point C $(\varphi=0)$

Fig. 10 Dimensionless stress intensity factor under the applied stress distribution expressed by Eq.(4),

$a / c \geq 1.0 \quad(a / t=0.2)$

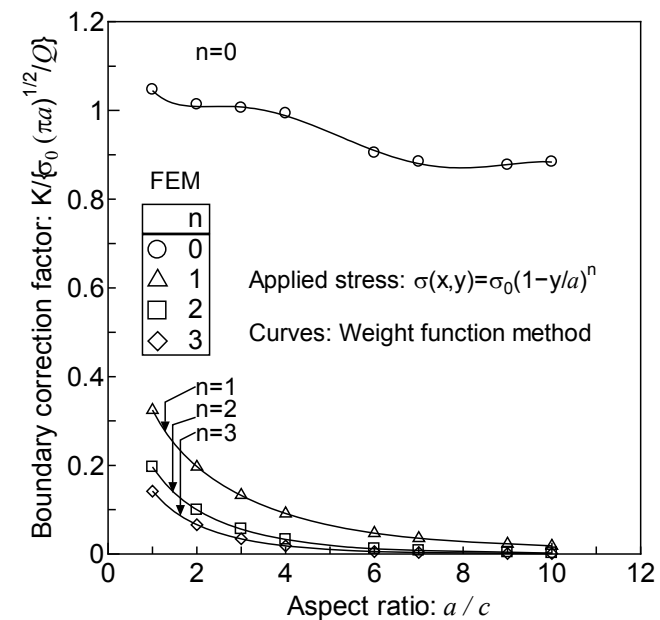

a) Point $\mathrm{A}(\varphi=\pi / 2)$

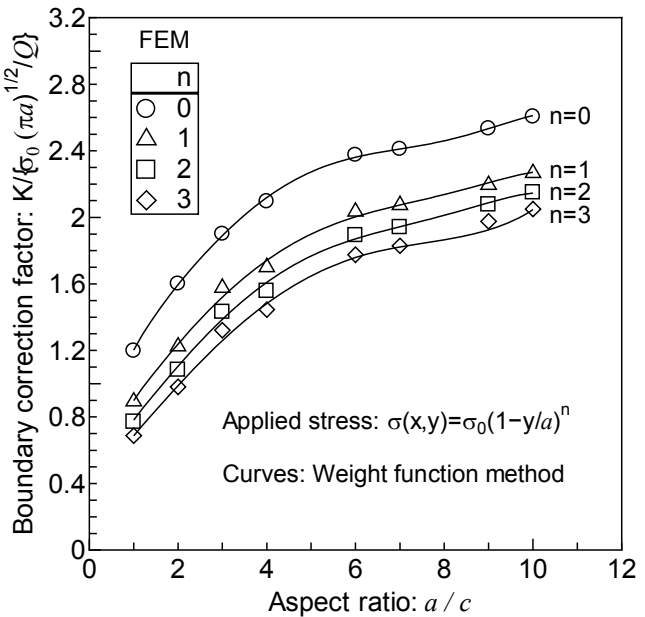

b) Point C $(\varphi=0)$ 
Fig. 11 Dimensionless stress intensity factor under the applied stress distribution expressed by Eq.(4),

$a / c \geq 1.0(a / t=0.4)$

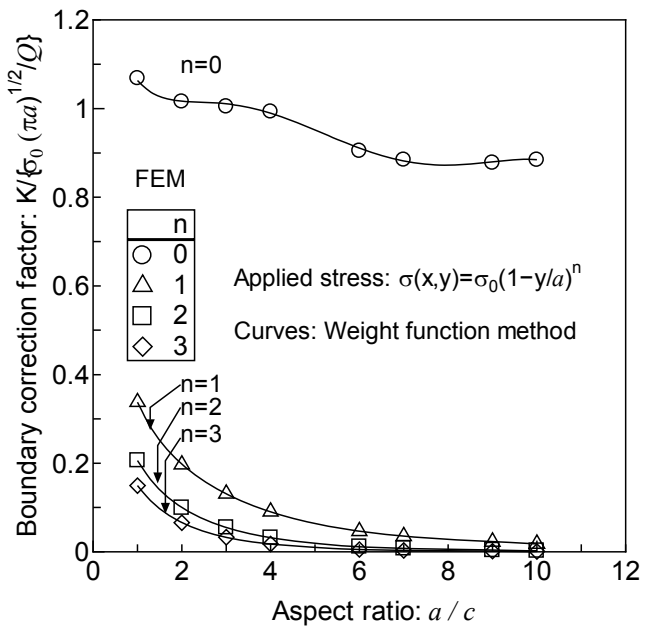

a) Point $\mathrm{A}(\varphi=\pi / 2)$

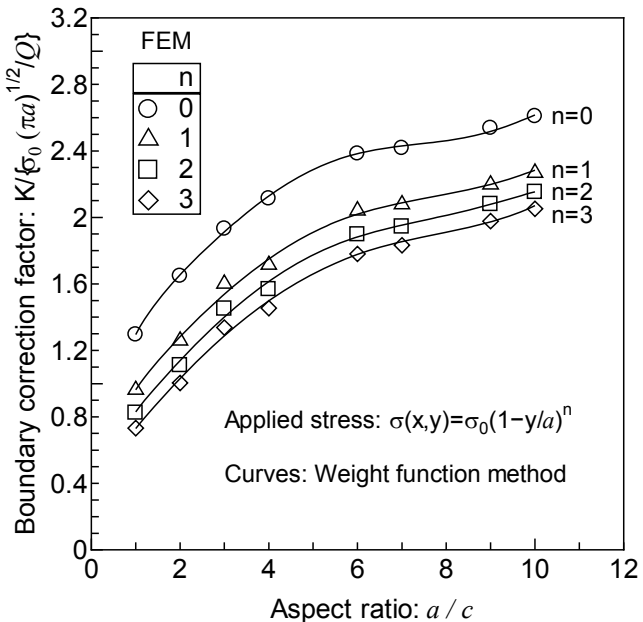

b) Point C $(\varphi=0)$

Fig. 12 Dimensionless stress intensity factor under the applied stress distribution expressed by Eq.(4),

$a / c \geq 1.0(a / t=0.6)$

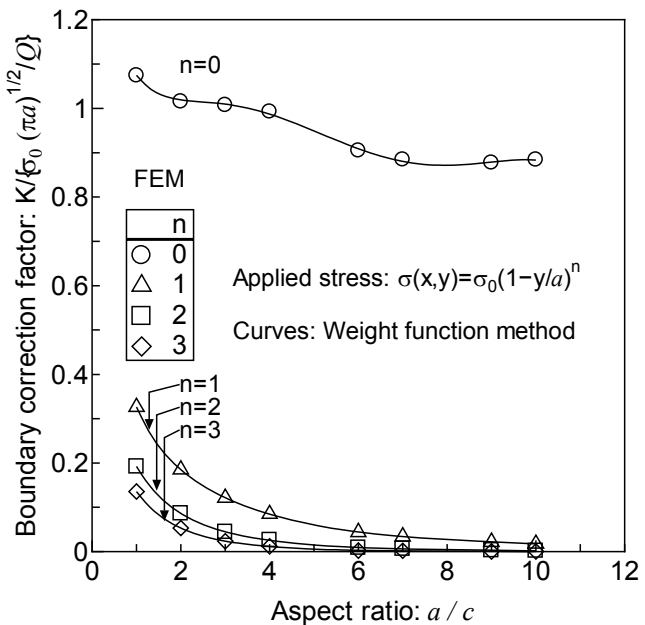

a) Point $\mathrm{A}(\varphi=\pi / 2)$

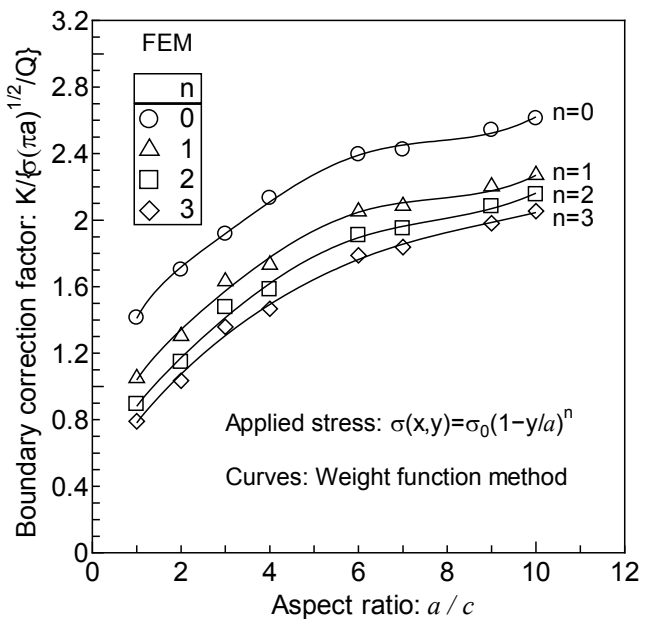

b) Point $\mathrm{C}(\varphi=0)$

Fig. 13 Dimensionless stress intensity factor under the applied stress distribution expressed by Eq.(4),

$a / c \geq 1.0 \quad(a / t=0.8)$ 


\section{Weight function for an embedded crack}

The verification of the weight function for the embedded crack shown in Fig. 14 is described in this section. The parameters to describe the crack geometries are shown in Fig. 14. The coordinate system for definition of the weight function is shown in Fig. 1. The verified crack shape had aspect ratios $a / c$ of $0.25,0.5$, 0.75 , and 1.0, the dimensionless shortest distance from the free surface to the crack $d / a$ was $0.25,0.4$, and 0.5 and the dimensionless crack width $c / b$ was 0.5 . Two cases where the distance from the crack front to the front and back of the plate was finite (plate thickness $t=2 d+2 a$ ) and infinite were verified. For the semi-infinite plate, the distance from the back free surface to point B was set to approximately $10 a$.

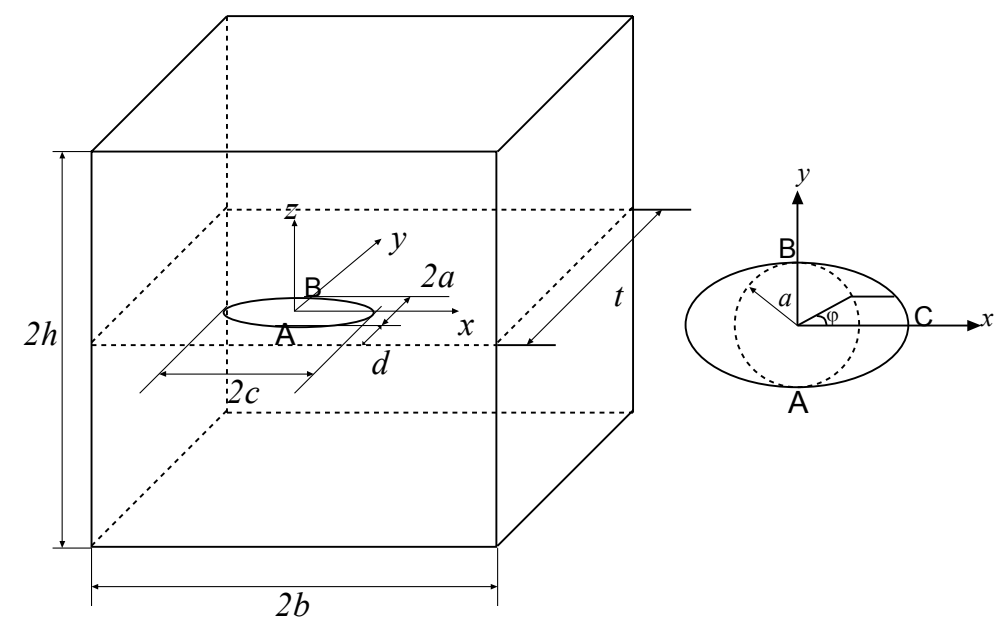

Fig. 14 Geometry of an embedded elliptical crack

Applied stress distributions on the crack face are expressed by Eqs. (6) and (7).

$$
\begin{aligned}
& \sigma(x, y)=\sigma_{0}(y / a)^{n},(n=0,1, \cdots 6) \\
& \sigma(x, y)=\sigma_{0}(x / c)^{n},(n=0,1, \cdots 6)
\end{aligned}
$$

In the embedded crack, the function coefficient $M$ cannot be expressed correctly by polynomial 
approximation of $\theta$ and $a / c$, so the dimensionless shortest distance from the free surface to the crack $d / a$ is also introduced as an additional variable to express $M$ as a polynomial approximation.

Figures 15 to 20 show the case where $d / a=0.25$ and Figs. 21 to 26 show the case where $d / a=0.5$. The relative error between stress intensity factors calculated with the weight function proposed in this paper and reference values increased when the crack shape was flat and the crack depth increased. The maximum error in the verification range was $9 \%$. It was confirmed that the weight function was applicable to an embedded crack in a semi-infinite and finite plates with a stress gradient in the direction of the thickness and width.

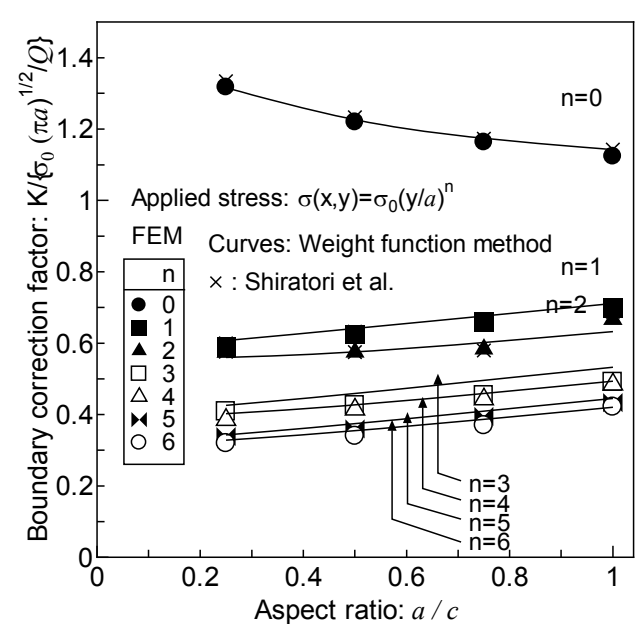

a) Semi-infinite body

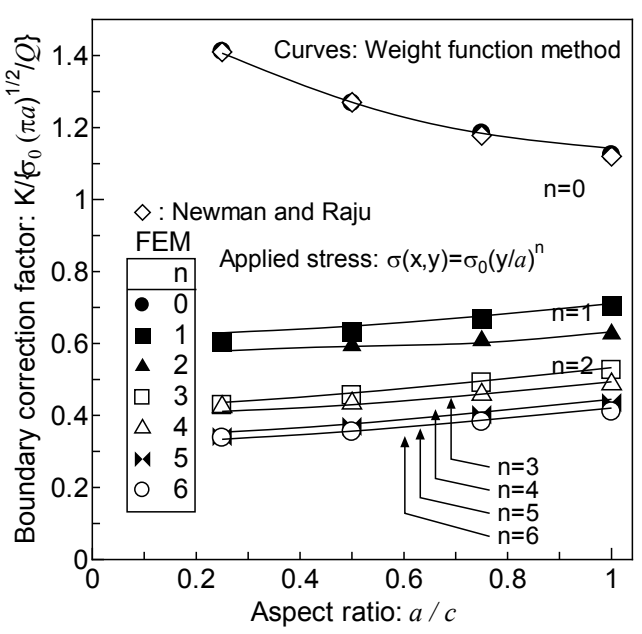

b) Finite body

Fig. 15. Dimensionless stress intensity factor at point $\mathrm{A}(\varphi=-\pi / 2)$ under the applied stress distribution expressed by Eq. (6) $(d / a=0.25)$ 


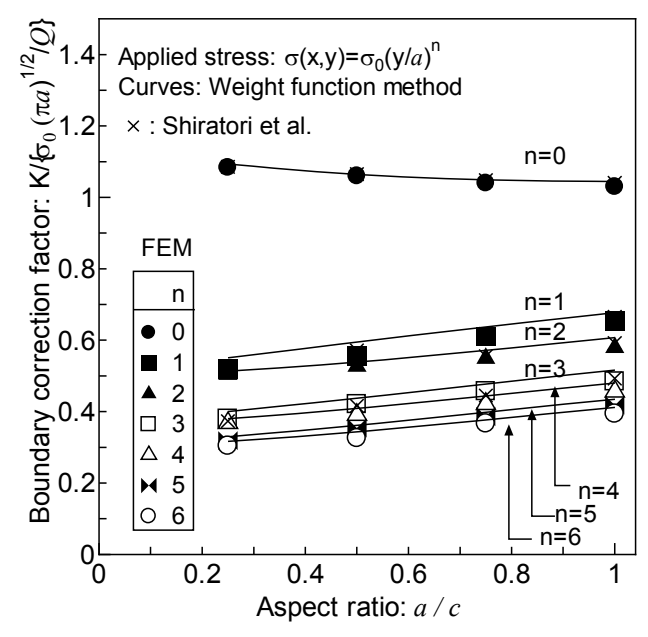

a) Semi-infinite body

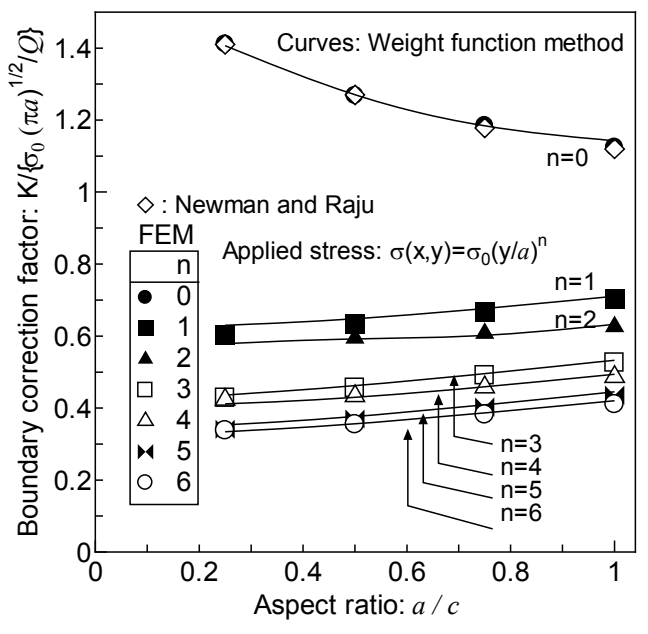

b) Finite body

Fig. 16. Dimensionless stress intensity factor at point $\mathrm{B}(\varphi=\pi / 2)$ under the applied stress distribution

expressed by Eq. (6) $(d / a=0.25)$

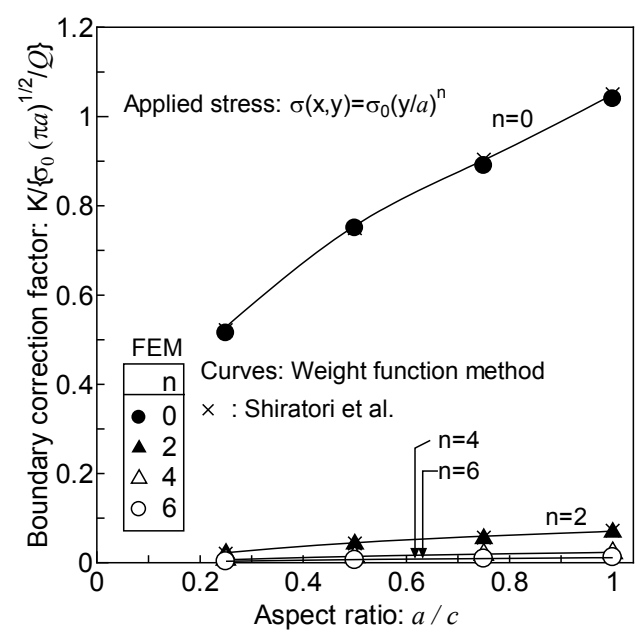

a) Semi-infinite body

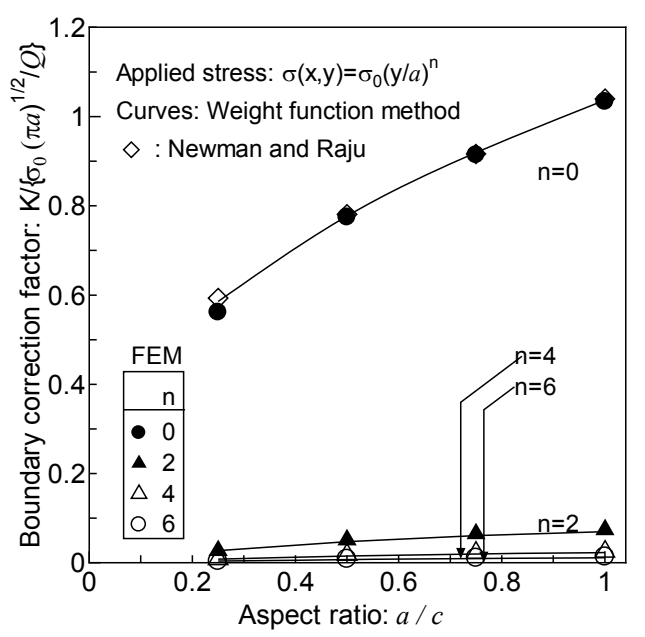

b) Finite body

Fig. 17. Dimensionless stress intensity factor at point $\mathrm{C}(\varphi=0)$ under the applied stress distribution expressed by Eq. (6) $(d / a=0.25)$ 


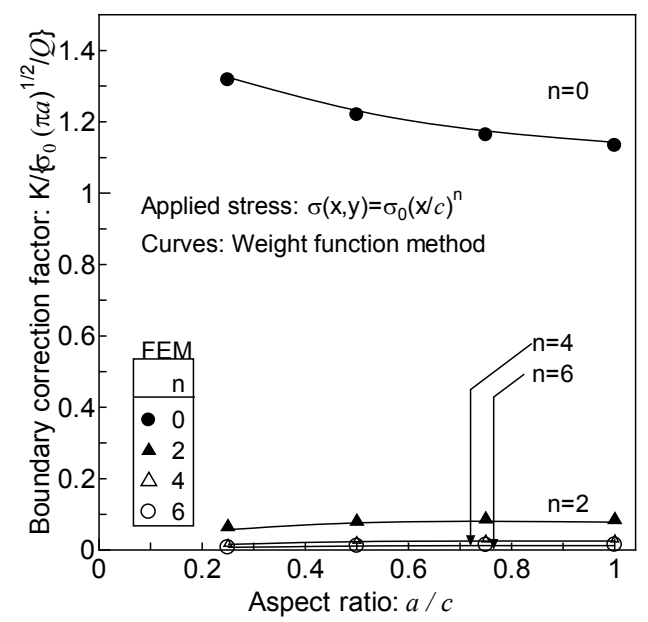

a) Semi-infinite body

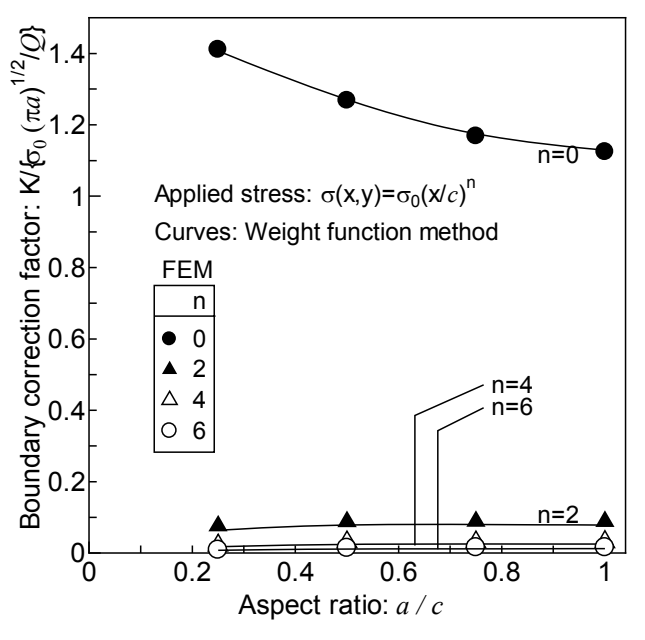

b) Finite body

Fig. 18. Dimensionless stress intensity factor at point $\mathrm{A}(\varphi=-\pi / 2)$ under the applied stress distribution expressed by Eq. (7) $(d / a=0.25)$

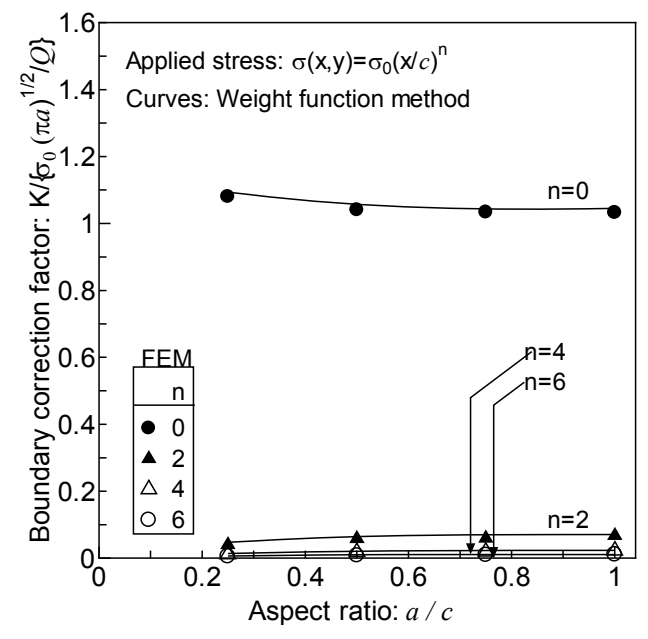

a) Semi-infinite body

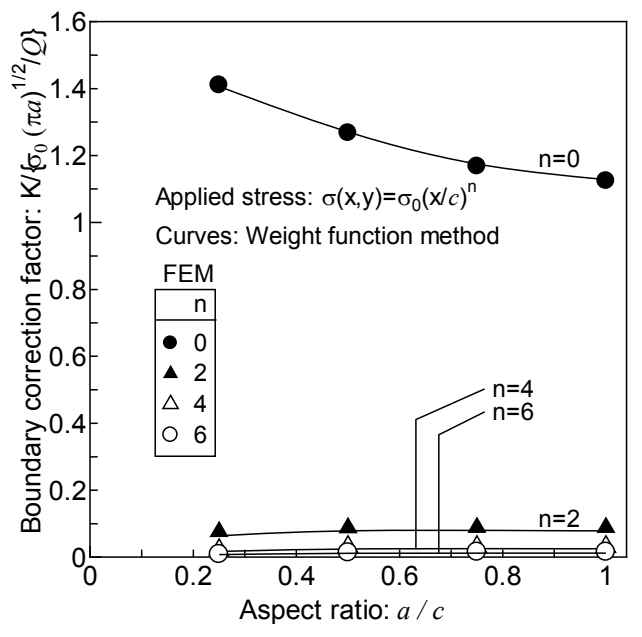

b) Finite body

Fig. 19. Dimensionless stress intensity factor at point $\mathrm{B}(\varphi=\pi / 2)$ under the applied stress distribution expressed by Eq. (7) $(d / a=0.25)$ 


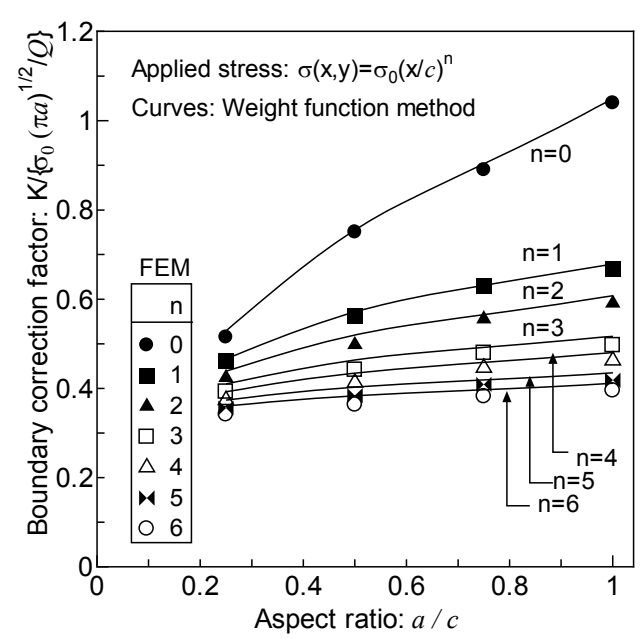

a) Semi-infinite body

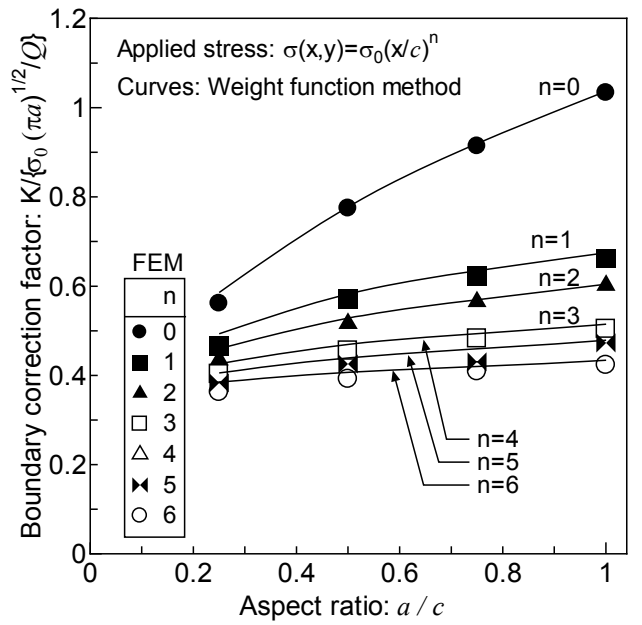

b) Finite body

Fig. 20. Dimensionless stress intensity factor at point $\mathrm{C}(\varphi=0)$ under the applied stress distribution

expressed by Eq. (7) $(d / a=0.25)$

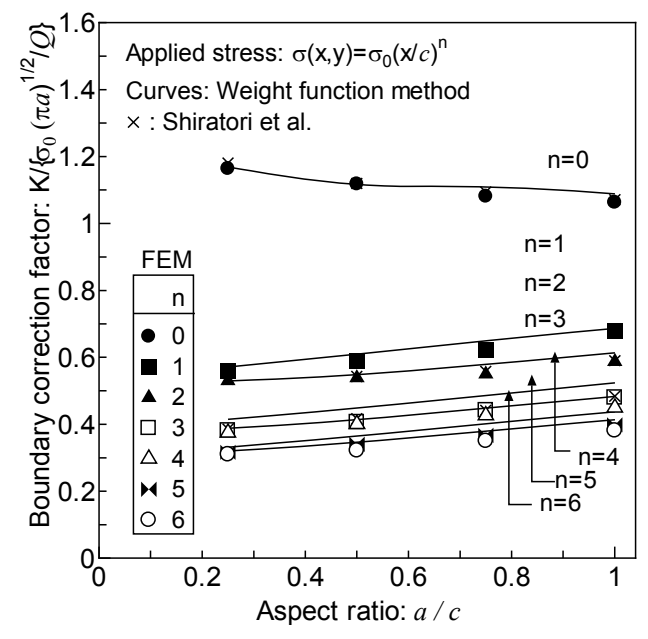

a) Semi-infinite body

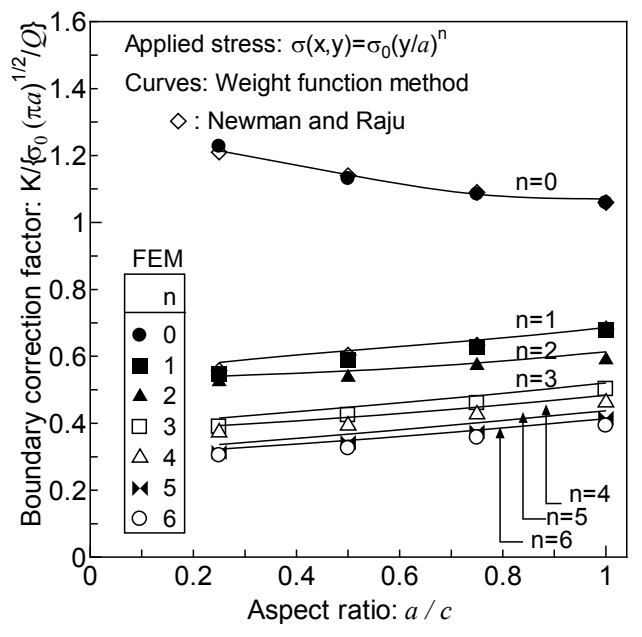

b) Finite body

Fig. 21. Dimensionless stress intensity factor at point $\mathrm{A}(\varphi=-\pi / 2)$ under the applied stress distribution expressed by Eq. (6) $(d / a=0.5)$ 


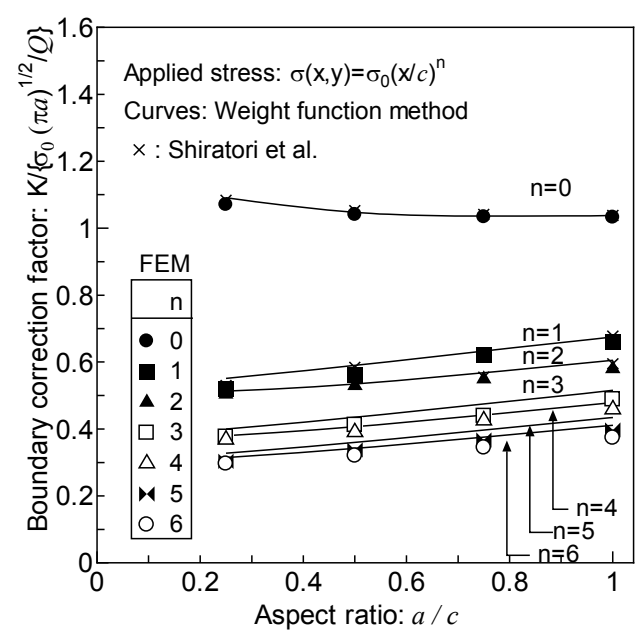

a) Semi-infinite body

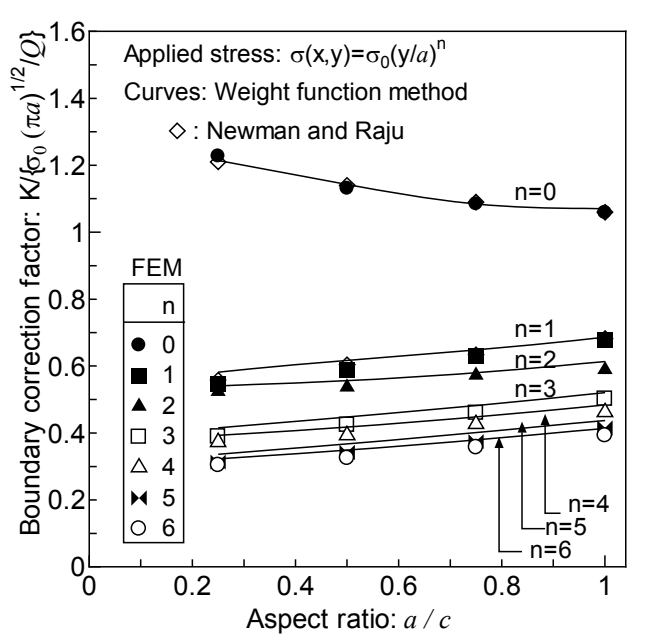

b) Finite body

Fig. 22. Dimensionless stress intensity factor at point B $(\varphi=\pi / 2)$ under the applied stress distribution expressed by Eq. (6) $(d / a=0.5)$

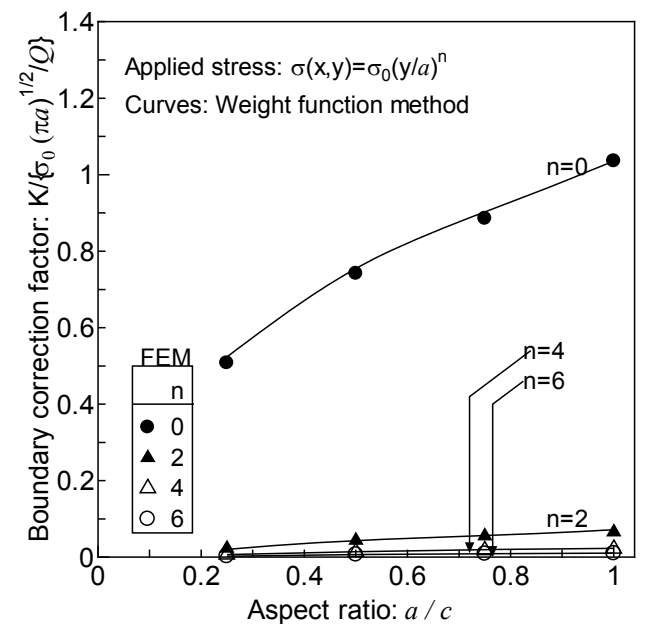

a) Semi-infinite body

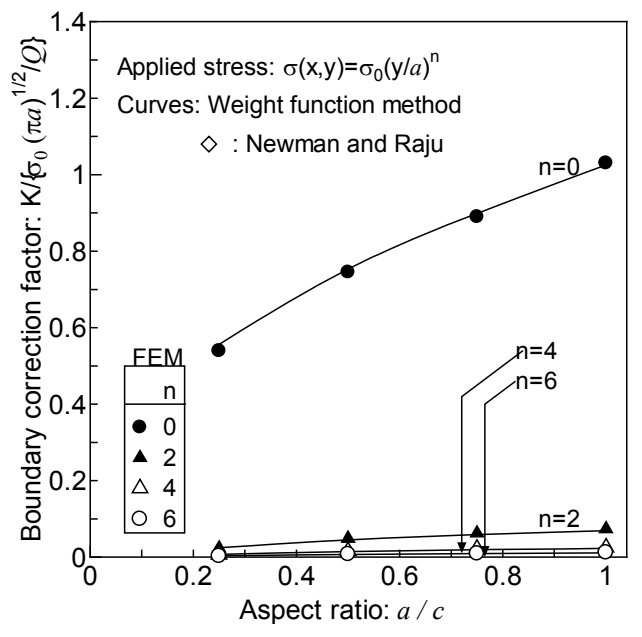

b) Finite body

Fig. 23. Dimensionless stress intensity factor at point $\mathrm{C}(\varphi=0)$ under the applied stress distribution expressed by Eq. (6) $(d / a=0.5)$ 


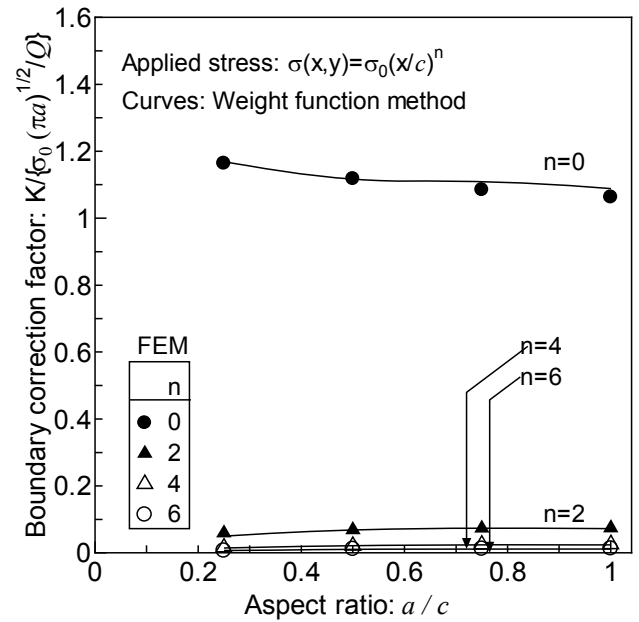

a) Semi-infinite body

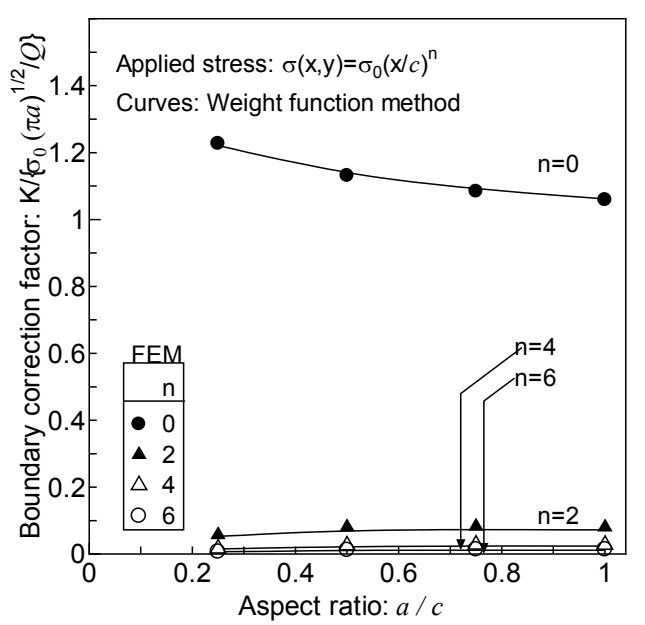

b) Finite body

Fig. 24. Dimensionless stress intensity factor at point $\mathrm{A}(\varphi=-\pi / 2)$ under the applied stress distribution expressed by Eq. (7) $(d / a=0.5)$

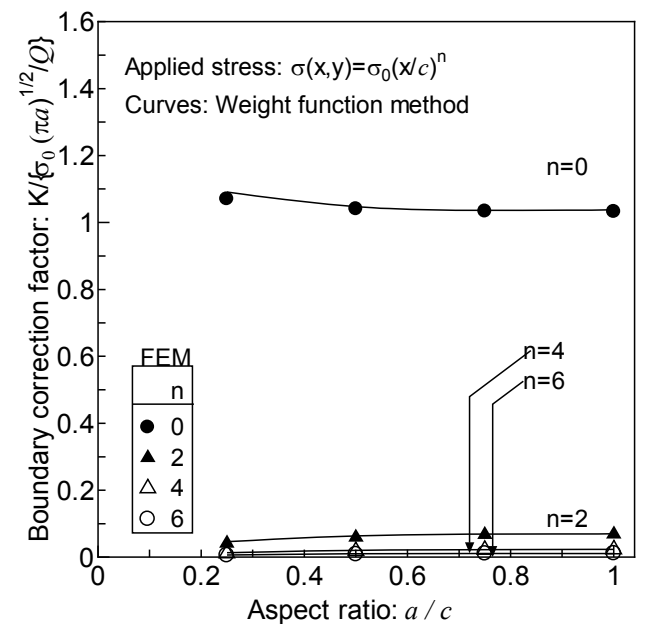

a) Semi-infinite body

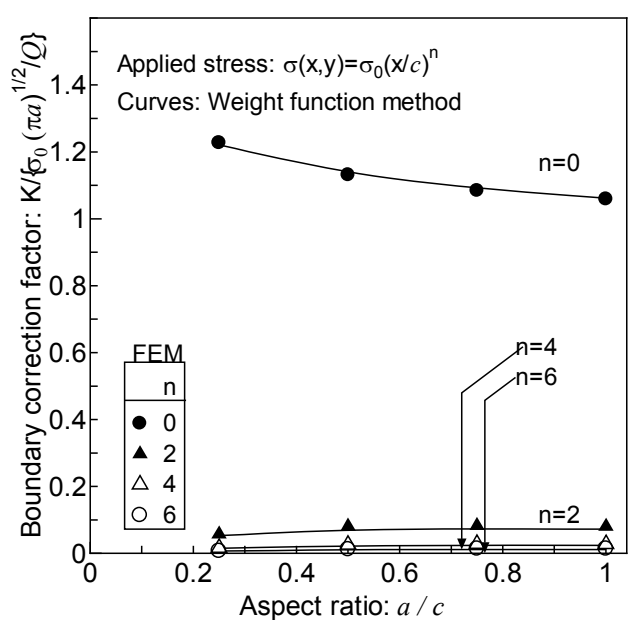

b) Finite body

Fig. 25. Dimensionless stress intensity factor at point B $(\varphi=\pi / 2)$ under the applied stress distribution expressed by Eq. (7) $(d / a=0.5)$ 


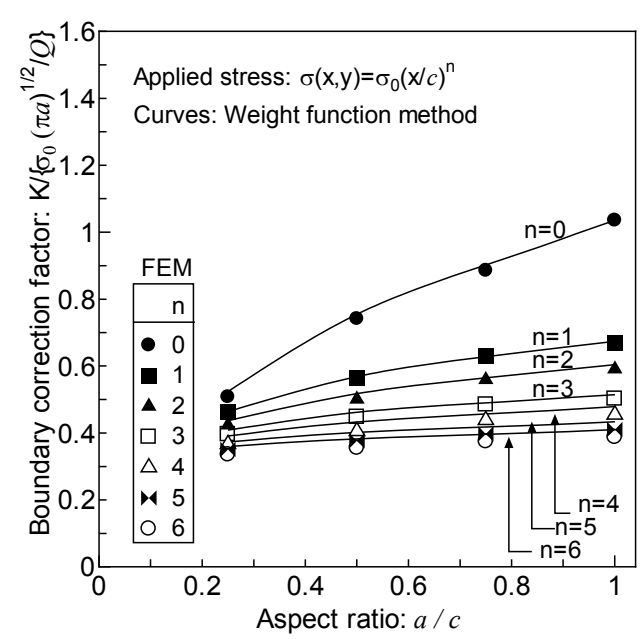

a) Semi-infinite body

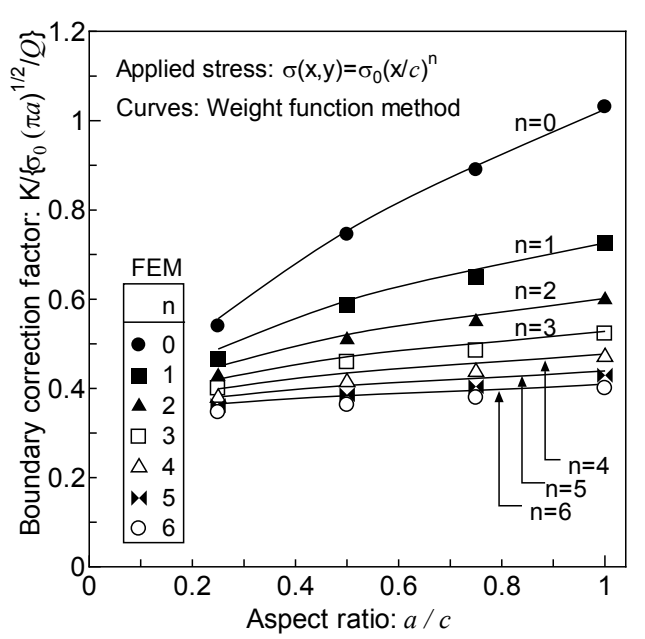

b) Finite body

Fig. 26. Dimensionless stress intensity factor at point $\mathrm{C}(\varphi=0)$ under the applied stress distribution expressed by Eq. (7) $(d / a=0.5)$

\section{Application to a surface and an embedded crack under complex stress field}

To verify the weight function of stress intensity factor proposed in this study extensively, the weight function was validated under a stress distribution in the direction of crack depth and width of the plate and under stress distribution applied on part of the crack surface.

\subsection{Stress field distributed in both directions of crack depth and width.}

The stress intensity factor under a stress field, which is described by Eq. (8) for a surface crack and Eq. (9) for an embedded crack, was verified. In this study, all combinations of $m=0-6$ and $n=0-6$ were verified.

$$
\begin{gathered}
\sigma(x, y)=\sigma_{0}(1-y / a)^{m}(1-x / c)^{n} \\
\sigma(x, y)=\sigma_{0}(y / a)^{m}(1-x / c)^{n}
\end{gathered}
$$




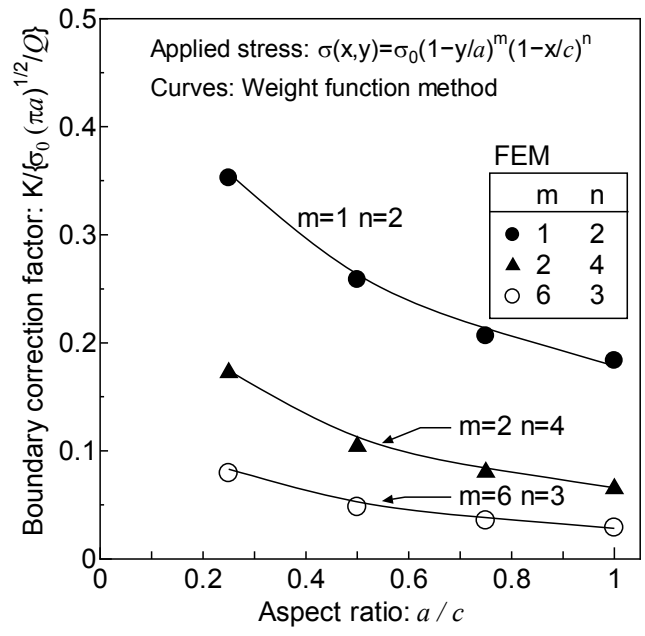

a) Point $\mathrm{A}(\varphi=\pi / 2)$

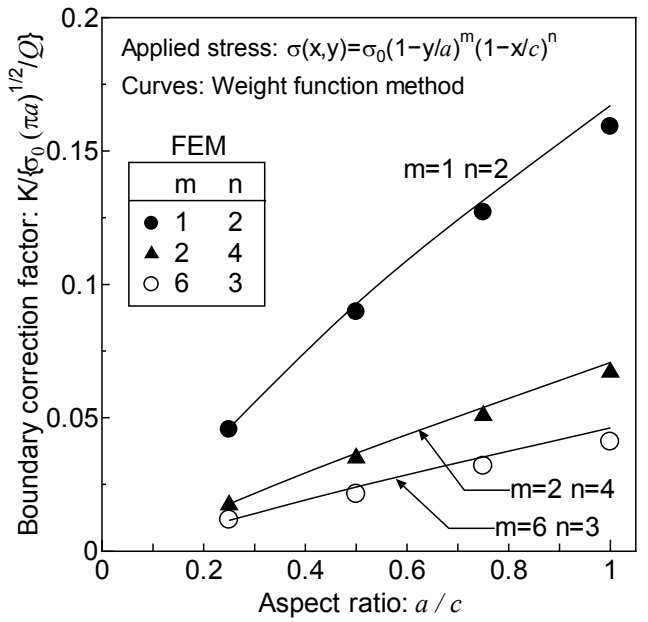

b) Point $\mathrm{C}(\varphi=0)$

Fig. 27 Dimensionless stress intensity factor on a surface crack under the applied stress distribution expressed by Eq.(8) $(a / t=0.3)$

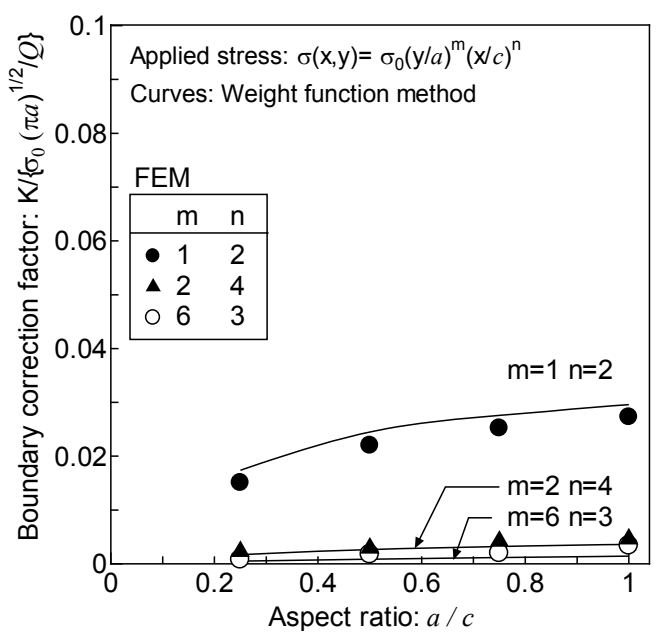

a) Point $\mathrm{A}(\varphi=\pi / 2)$

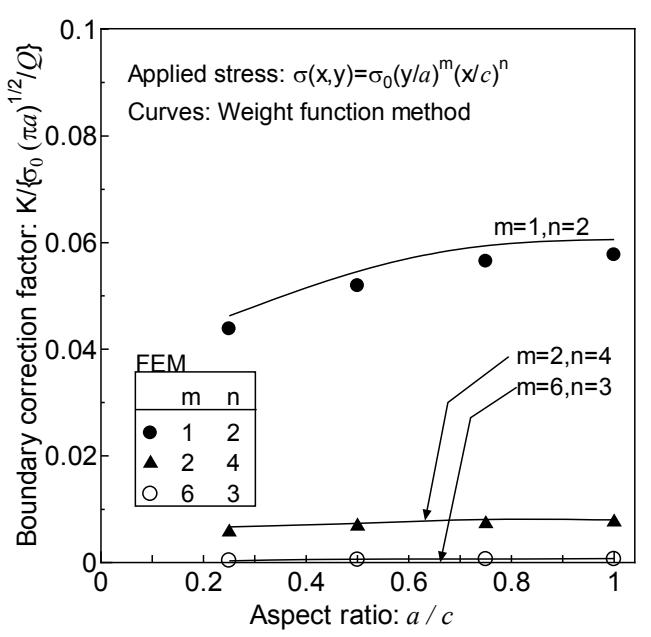

b) Point $\mathrm{C}(\varphi=0)$

Fig. 28 Dimensionless stress intensity factor in finite thickness cracked body under the applied stress distribution expressed by Eq.(9) $(d / a=0.25)$

Figures 27 and 28 show the validation results for the stress fields of $(m=1, n=2),(m=2, n=4)$, and $(m=6$, 
$n=3$ ). These figures confirm that if the weight function proposed in this study is applied to the stress field in the direction of crack depth and width, the stress intensity factor can be calculated with good accuracy. Under other verified conditions, the accuracy of the estimation is comparable.

\subsection{Stress field distributed on part of the crack face.}

The stress intensity factor was verified under a stress distribution applied to part of the crack surface for a surface crack and an embedded crack. The verification was conducted under the constant stress defined by Eq. (10), which was applied to part of the crack face in the direction of the crack depth. Next, the verification was conducted under the constant stress defined as Eq. (11), which was applied to part of the crack at the surface.

$$
\begin{gathered}
\sigma(x, y)=\sigma_{0},(-a / 4 \leq y \leq a / 4) \\
\sigma(x, y)=\sigma_{0},(-a / 4 \leq x \leq a / 4,-a / 4 \leq y \leq a / 4)
\end{gathered}
$$

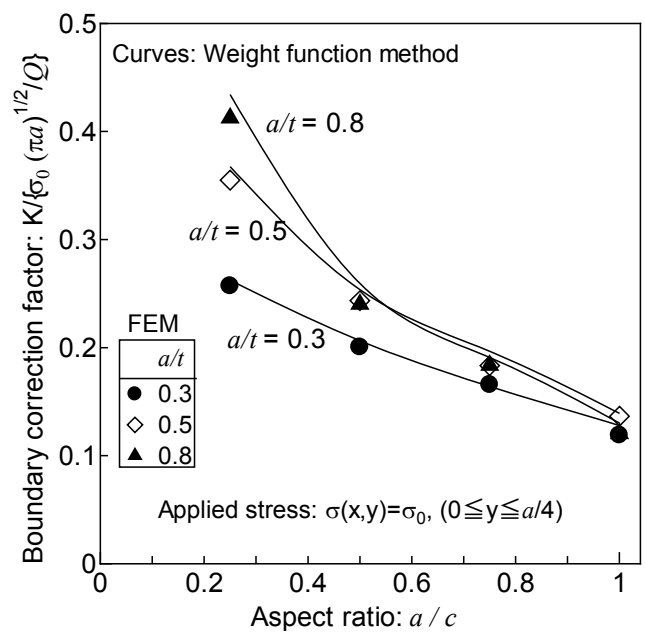

a) Point $\mathrm{A}(\varphi=\pi / 2)$

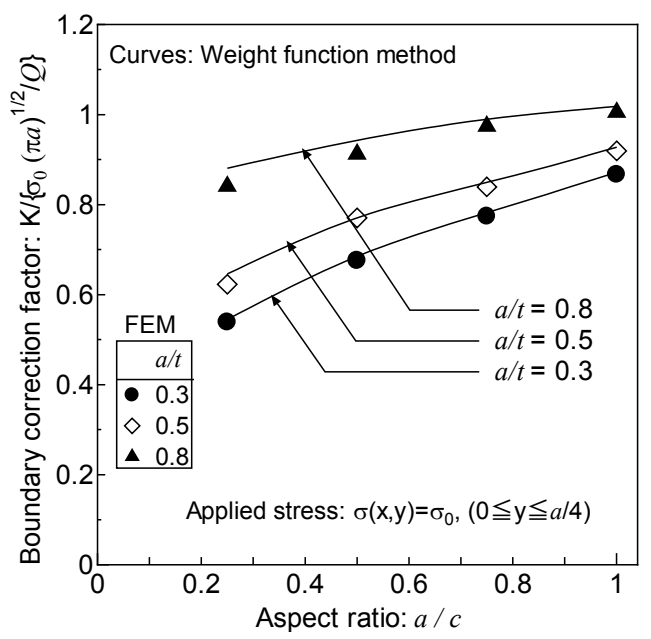

b) Point $\mathrm{C}(\varphi=0)$

Fig. 29 Dimensionless stress intensity factor on surface crack under the applied stress distribution expressed by Eq. (10) 


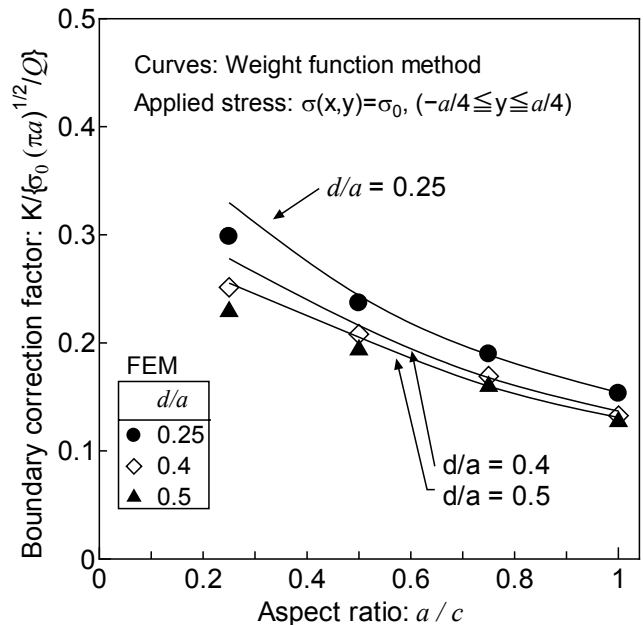

a) Point $\mathrm{A}(\varphi=\pi / 2)$

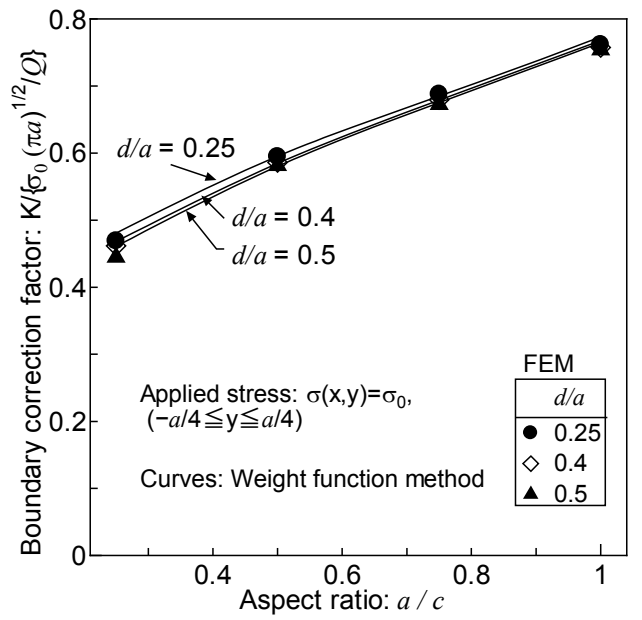

b) Point $\mathrm{C}(\varphi=0)$

Fig. 30 Dimensionless stress intensity factor in crack embedded in body with finite thickness under the applied stress distribution expressed by Eq. (10)

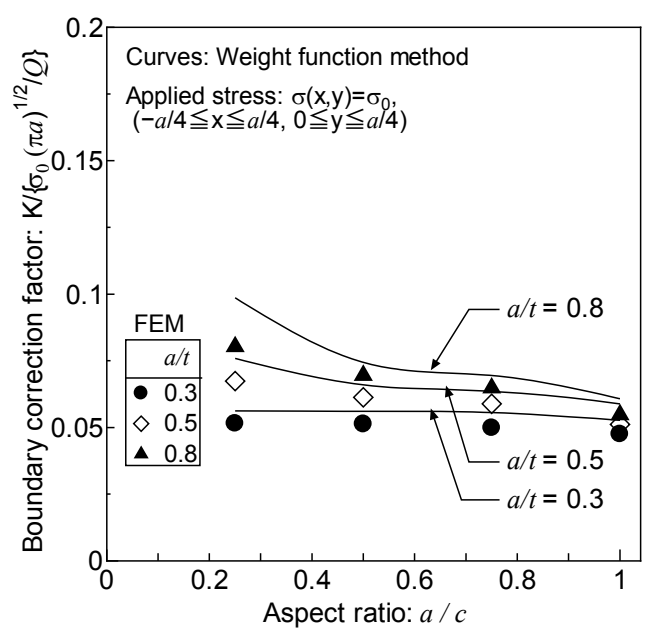

a) Point $\mathrm{A}(\varphi=\pi / 2)$

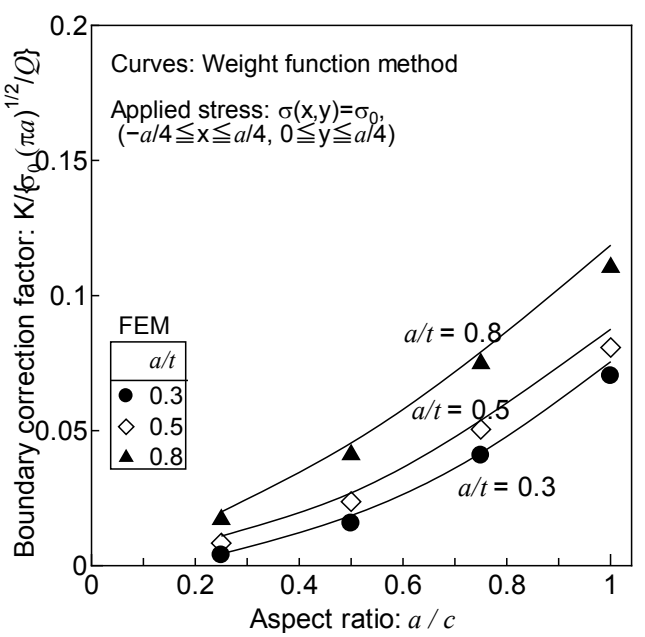

b) Point $\mathrm{C}(\varphi=0)$

Fig. 31 Dimensionless stress intensity factor on surface crack under the applied stress distribution expressed by Eq. (11) 


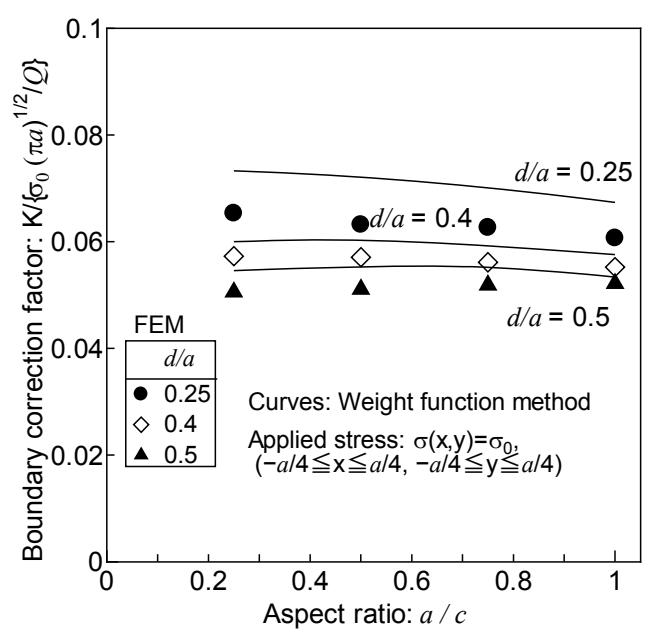

a) Point $\mathrm{A}(\varphi=\pi / 2)$

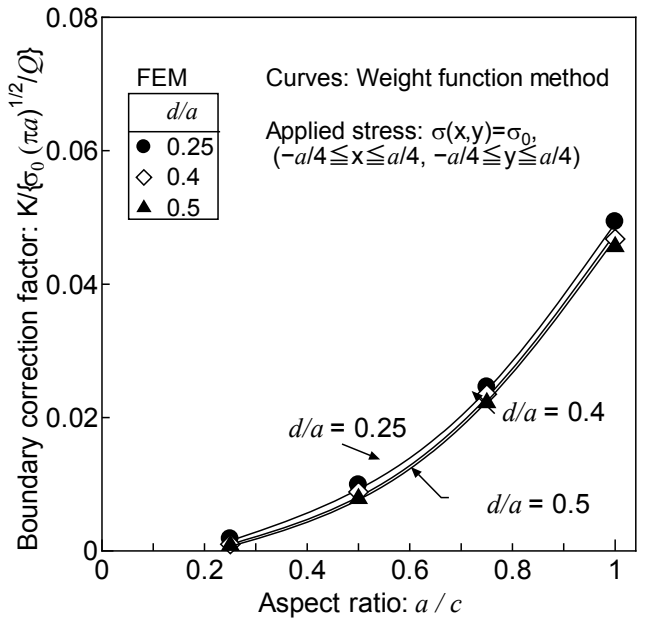

b) Point $\mathrm{C}(\varphi=0)$

Fig. 32 Dimensionless stress intensity factor in crack embedded in body with finite thickness under the applied stress distribution expressed by Eq. (11)

Figures 29 to 32 show the validation results for each stress field. These figures confirm that the accuracy of the estimation tends to be inferior when the edge of the crack is closer to the free surface. The reason why is that the number of finite element mesh divisions between the crack front and free surface might be insufficient to express the crack front deformation behavior. The coefficient of weight function can be derived by polynomial approximation. If the allowable error between the reference solution and the stress intensity factor calculated by the weight function is less than $10 \%$, the weight function is considered to produce an improved solution under the stress field applied to part of the crack surface

\section{Conclusions}

The weight function for a surface crack and an embedded crack proposed by Wang and Glinka was improved for a wider stress field and more diverse crack shapes. The weight function was improved to be applicable to a crack embedded in a plate with finite thickness. The application range of the weight function 
proposed in this study is given as follows if the allowable error of stress intensity factor is $10 \%$ or less:

- $\quad$ A surface crack: $0<a / c \leq 10,0<a / t<1, c / b \leq 0.5$.

- $\quad$ An embedded crack: $0<a / c \leq 1,0.25<d / a<0.5, c / b \leq 0.5$. 


\section{Appendix: Polynomial approximation of coefficient of the weight function $M$}

The polynomial approximation of the coefficient of the weight function $M$ is shown in this appendix for each crack morphology, where $\alpha=a / c$ and $\zeta=a / t$.

A.1 Surface crack $(0<\alpha \leq 1.0)$

A.1.1 Deepest point (point $\mathrm{A}$ in Fig. $1 ; \varphi=\pi / 2$ )

$$
\begin{aligned}
& M=B_{1} \zeta^{6}+B_{2} \zeta^{5}+B_{3} \zeta^{4}+B_{4} \zeta^{3}+B_{5} \zeta^{2}+B_{6} \zeta \\
& B_{1}=A_{11} \alpha^{4}+A_{12} \alpha^{3}+A_{13} \alpha^{2}+A_{14} \alpha+A_{15}(\alpha+0.15)^{-1}+A_{16} \\
& B_{2}=A_{21} \alpha^{4}+A_{22} \alpha^{3}+A_{23} \alpha^{2}+A_{24} \alpha+A_{25}(\alpha+0.15)^{-1}+A_{26} \\
& B_{3}=A_{31} \alpha^{4}+A_{32} \alpha^{3}+A_{33} \alpha^{2}+A_{34} \alpha+A_{35}(\alpha+0.15)^{-1}+A_{36} \\
& B_{4}=A_{41} \alpha^{4}+A_{42} \alpha^{3}+A_{43} \alpha^{2}+A_{44} \alpha+A_{45}(\alpha+0.15)^{-1}+A_{46} \\
& B_{5}=A_{51} \alpha^{4}+A_{52} \alpha^{3}+A_{53} \alpha^{2}+A_{54} \alpha+A_{55}(\alpha+0.15)^{-1}+A_{56} \\
& B_{6}=A_{61} \alpha^{4}+A_{62} \alpha^{3}+A_{63} \alpha^{2}+A_{64} \alpha+A_{65}(\alpha+0.15)^{-1}+A_{66} \\
& A_{i j}=\left[\begin{array}{cccccc}
-60.964 & 57.038 & -31.942 & 101.36 & 37.976 & 8.5477 \\
50.931 & 30.096 & -64.030 & -14.159 & -32.751 & -198.97 \\
3.7590 & 96.704 & -239.63 & 37.624 & -18.744 & 294.32 \\
-97.234 & 24.657 & 143.31 & 35.240 & 35.472 & -206.31 \\
90.445 & -152.37 & 112.43 & -100.35 & -14.417 & 78.789 \\
-39.038 & 98.466 & -102.92 & 57.458 & 3.7526 & -19.082
\end{array}\right]
\end{aligned}
$$


A.1.2 Surface point (point $C$ in Fig. $1 ; \varphi=0$ )

$$
\begin{aligned}
& M=B_{1} \zeta^{6}+B_{2} \zeta^{5}+B_{3} \zeta^{4}+B_{4} \zeta^{3}+B_{5} \zeta^{2}+B_{6} \zeta \\
& B_{1}=A_{11} \alpha^{4}+A_{12} \alpha^{3}+A_{13} \alpha^{2}+A_{14} \alpha+A_{15} \alpha^{-1}+A_{16} \\
& B_{2}=A_{21} \alpha^{4}+A_{22} \alpha^{3}+A_{23} \alpha^{2}+A_{24} \alpha+A_{25} \alpha^{-1}+A_{26} \\
& B_{3}=A_{31} \alpha^{4}+A_{32} \alpha^{3}+A_{33} \alpha^{2}+A_{34}(\alpha+0.1)+A_{35}(\alpha+0.1)^{-1}+A_{36} \\
& B_{4}=A_{41} \alpha^{4}+A_{42} \alpha^{3}+A_{43} \alpha^{2}+A_{44}(\alpha+0.1)+A_{45}(\alpha+0.1)^{-1}+A_{46} \\
& B_{5}=A_{51} \alpha^{4}+A_{52} \alpha^{3}+A_{53} \alpha^{2}+A_{54}(\alpha+0.1)+A_{55}(\alpha+0.1)^{-1}+A_{56} \\
& B_{6}=A_{61} \alpha^{4}+A_{62} \alpha^{3}+A_{63} \alpha^{2}+A_{64}(\alpha+0.1)+A_{65}(\alpha+0.1)^{-1}+A_{66} \\
& A_{i j}=\left[\begin{array}{cccccc}
46270 & -141360 & 157780 & -73619 & -52.740 & 12240 \\
-138420 & 410340 & -441890 & 198070 & 47.100 & -31160 \\
150810 & -436180 & 454800 & -194860 & 511.10 & 47179 \\
-74872 & -212000 & -214490 & 87290 & -693.20 & -19205 \\
17248 & -48052 & 47482 & -18474 & 256.20 & 3649.1 \\
-1525.0 & 4214.0 & -4114.0 & 1563.0 & -27.500 & -284.50
\end{array}\right]
\end{aligned}
$$


A.2 Surface crack $(1.0<a / c \leq 10)$

A.2.1 Deepest point (point A in Fig. 1; $\varphi=\pi / 2$ )

A.2.1.1 $n=0$

$$
\begin{gathered}
M=B_{5} \zeta^{3}+B_{6} \zeta^{2}+B_{7} \zeta+B_{8} \\
B_{5}=A_{58} \\
B_{6}=A_{68} \\
B_{7}=A_{75} \alpha^{3}+A_{76} \alpha^{2}+A_{77} \alpha \\
A_{i j}=\left[\begin{array}{ccccccc}
0 \\
0
\end{array}\right] \\
0
\end{gathered}
$$


A.2.1.2 $n=1$

$$
\begin{gathered}
M=B_{4} \zeta^{4}+B_{5} \zeta^{3}+B_{6} \zeta^{2}+B_{7} \zeta+B_{8} \\
B_{4}=A_{46} \alpha^{2}+A_{48} \\
B_{5}=A_{55} \alpha^{3}+A_{56} \alpha^{2}+A_{57} \alpha+A_{58} \\
B_{6}=A_{64} \alpha^{4}+A_{65} \alpha^{3}+A_{67} \alpha \\
A_{i j}=A_{72} \alpha^{6}+A_{73} \alpha^{5}+A_{74} \alpha^{4}+A_{75} \alpha^{3}+A_{76} \alpha^{2}+A_{78} \\
0 \\
B_{8}=A_{82} \alpha^{6}+A_{83} \alpha^{5}+A_{84} \alpha^{4}+A_{87} \alpha+A_{88} \\
0 \\
0
\end{gathered}
$$


A.2.1.3 $n=2$

$$
\begin{aligned}
& M=B_{3} \zeta^{5}+B_{4} \zeta^{4}+B_{5} \zeta^{3}+B_{6} \zeta^{2}+B_{7} \zeta+B_{8} \\
& B_{3}=A_{37} \alpha+A_{38} \\
& B_{4}=A_{46} \alpha^{2} \\
& B_{5}=A_{56} \alpha^{2} \\
& B_{6}=A_{64} \alpha^{4}+A_{65} \alpha^{3}+A_{66} \alpha^{2}+A_{67} \alpha+A_{68} \\
& B_{7}=A_{73} \alpha^{5}+A_{74} \alpha^{4}+A_{75} \alpha^{3} \\
& B_{8}=A_{81} \alpha^{7}+A_{82} \alpha^{6}+A_{83} \alpha^{5}+A_{84} \alpha^{4}+A_{85} \alpha^{3}+A_{86} \alpha^{2}+A_{88} \\
& A_{i j}=\left[\begin{array}{cccccccc}
0 & 0 & 0 & 0 & 0 & 0 & 0 & 0 \\
0 & 0 & 0 & 0 & 0 & 0 & 0 & 0 \\
0 & 0 & 0 & 0 & 0 & 0 & 0.49114 & -2.2355 \\
0 & 0 & 0 & 0 & 0 & 0.15053 & 0 & 0 \\
0 & 0 & 0 & 0 & 0 & -0.37596 & 0 & 0 \\
0 & 0 & 0 & 2.2185 \times 10^{-3} & -0.054023 & 0.75293 & -1.7700 & 2.3917 \\
0 & 0 & -1.9326 \times 10^{-4} & 4.3894 \times 10^{-3} & -0.032880 & 0 & 0 & 0 \\
9.19829 \times 10^{-6} & -3.4073 \times 10^{-4} & 5.1860 \times 10^{-3} & -0.042239 & 0.19751 & -0.46542 & 0 & 0.22384
\end{array}\right]
\end{aligned}
$$


A.2.1.4 $n=3$

$$
\begin{gathered}
M=B_{3} \zeta^{5}+B_{4} \zeta^{4}+B_{5} \zeta^{3}+B_{6} \zeta^{2}+B_{7} \zeta+B_{8} \\
B_{3}=A_{37} \alpha \\
B_{4}=A_{48} \\
B_{5}=A_{56} \alpha^{2}+A_{57} \alpha \\
B_{6}=A_{64} \alpha^{4}+A_{65} \alpha^{3}+A_{66} \alpha^{2}+A_{67} \alpha+A_{68} \\
A_{i j}= \\
0 \\
B_{8}=A_{81} \alpha^{7}+A_{82} \alpha^{6}+A_{83} \alpha^{5}+A_{84} \alpha^{4}+A_{85} \alpha^{3}+A_{86} \alpha^{2}+A_{88} \\
0 \\
0
\end{gathered}
$$


A.2.2 Surface point (point $C$ in Fig. $2 ; \varphi=0$ )

A.2.2.1 $n=0$

$$
\begin{aligned}
& M=B_{3} \zeta^{5}+B_{4} \zeta^{4}+B_{5} \zeta^{3}+B_{6} \zeta^{2}+B_{7} \zeta+B_{8} \\
& B_{3}=A_{38} \\
& B_{4}=A_{47} \alpha+A_{48} \\
& B_{5}=A_{57} \alpha+A_{58} \\
& B_{6}=A_{55} \alpha^{3}+A_{56} \alpha^{2} \\
& B_{7}=A_{74} \alpha^{4}+A_{75} \alpha^{3}+A_{76} \alpha^{2}+A_{77} \alpha \\
& B_{8}=A_{83} \alpha^{5}+A_{84} \alpha^{4}+A_{85} \alpha^{3}+A_{86} \alpha^{2}+A_{88} \\
& A_{i j}=\left[\begin{array}{cccccccc}
0.43567 & 0 & 0 & 22.680 & -38.142 & 19.994 & 0 & 0 \\
0 & -1.5490 & 0 & -2.8220 & 1.5087 & 0 & 0 & 0 \\
0.052314 & 0.42567 & 0.31897 & 0 & 0 & 0 & 0 & 0 \\
-0.025980 & -0.063798 & -0.016804 & 0 & 0 & 0 & 0 & 0 \\
3.6182 \times 10^{-3} & 3.1098 \times 10^{-3} & 0 & 0 & 0 & 0 & 0 & 0 \\
-1.5929 \times 10^{-4} & 0 & 0 & 0 & 0 & 0 & 0 & 0 \\
0 & 0 & 0 & 0 & 0 & 0 & 0 & 0 \\
0 & 0 & 0 & 0 & 0 & 0 & 0 & 0
\end{array}\right]
\end{aligned}
$$


A.2.2.2 $n=1$

$$
\begin{gathered}
M=B_{3} \zeta^{5}+B_{4} \zeta^{4}+B_{5} \zeta^{3}+B_{6} \zeta^{2}+B_{7} \zeta+B_{8} \\
B_{3}=A_{38} \\
B_{4}=A_{48} \\
B_{5}=A_{58} \\
B_{6}=A_{67} \alpha \\
A_{i j}= \\
{\left[\begin{array}{ccccccc}
0 \\
0
\end{array}\right]} \\
0 \\
0
\end{gathered}
$$


A.2.2.3 $n=2$

$$
\begin{gathered}
M=B_{7} \zeta+B_{8} \\
B_{7}=A_{74} \alpha^{4}+A_{75} \alpha^{3}+A_{76} \alpha^{2}+A_{77} \alpha+A_{78} \\
B_{i j}=A_{83} \alpha^{5}+A_{84} \alpha^{4}+A_{86} \alpha^{2}+A_{87} \alpha+A_{88} \\
=\left[\begin{array}{cccccccc}
0 & 0 & 0 & 0 & 0 & 0 & 0 & 0 \\
0 & 0 & 0 & 0 & 0 & 0 & 0 & 0 \\
0 & 0 & 0 & 0 & 0 & 0 & 0 & 0 \\
0 & 0 & 0 & 0 & 0 & 0 & 0 & 0 \\
0 & 0 & 0 & 0 & 0 & 0 & 0 & 0 \\
0 & 0 & 0 & 0 & 0 & 0 & 0 & 0 \\
0 & 0 & 0 & 2.4662 \times 10^{-3} & -0.063952 & 0.59367 & -2.3420 & 3.3807 \\
0 & 0 & -1.0784 \times 10^{-4} & 1.8976 \times 10^{-3} & 0 & -0.15994 & 0.82173 & -1.2159
\end{array}\right]
\end{gathered}
$$

A.2.2.4 $n=3$

$$
\begin{gathered}
M=B_{4} \zeta^{4}+B_{5} \zeta^{3}+B_{6} \zeta^{2}+B_{8} \\
B_{4}=A_{48} \\
B_{5}=A_{58} \\
B_{6}=A_{65} \alpha^{3}+A_{66} \alpha^{2}+A_{67} \alpha \\
A_{i j}=A_{84} \alpha^{4}+A_{86} \alpha^{2}+A_{87} \alpha+A_{88} \\
{\left[\begin{array}{cccccccc}
0 & 0 & 0 & 0 & 0 & 0 & 0 & 0 \\
0 & 0 & 0 & 0 & 0 & 0 & 0 & 0 \\
0 & 0 & 0 & 0 & 0 & 0 & 0 & 0 \\
0 & 0 & 0 & 0 & 0 & 0 & 0 & -4.5556 \\
0 & 0 & 0 & 0 & 0 & 0 & 0 & 6.6152 \\
0 & 0 & 0 & 0 & -9.8715 \times 10^{-3} & 0.19928 & -1.2588 & 0 \\
0 & 0 & 0 & 0 & 0 & 0 & 0 & 0 \\
0 & 0 & 0 & 2.3419 \times 10^{-4} & 0 & -0.058428 & 0.32548 & -0.45246
\end{array}\right]}
\end{gathered}
$$




\section{A.3 Embedded crack}

\section{A.3.1 Finite plate thickness}

The coefficient of weight function $M$ at the ellipse vertex position inside and outside the plate (corresponding to point A in Fig. 1) is given as a polynomial approximation, where $\eta=d / a$ and $\alpha=a / c$.

$$
\begin{aligned}
& M=C_{1}+C_{2} \alpha+C_{3} \eta+C_{4} \alpha^{-0.5}+C_{5} \eta^{0.5}+C_{6} \alpha^{0.5} \eta+C_{7} \alpha^{0.6} \\
& C_{1}=6.3517, C_{2}=1.9054, C_{3}=1.5872, C_{4}=-0.55225, \\
& C_{5}=-5.9005, C_{6}=2.0248, C_{7}=-5.3093
\end{aligned}
$$

The coefficient of weight function $M$ at the ellipse vertex position in the plate width direction (corresponding to point $\mathrm{C}$ in Fig. 1) is given as an polynomial approximation, where $\eta=d / a$ and $\alpha=a / c$.

$$
\begin{gathered}
M=D_{1}+D_{2} \alpha^{-1}+D_{3} \eta^{-1}+D_{4} \alpha^{-0.5}+D_{5} \eta^{-2}+D_{6} \alpha^{-0.5} \eta^{-2}+D_{7} \alpha^{-0.5} \eta^{-1} \\
D_{1}=0.47163, D_{2}=-0.41006, D_{3}=-0.50356, D_{4}=-0.20652, \\
D_{5}=0.057275, D_{6}=-0.056419, D_{7}=0.51349
\end{gathered}
$$

\section{A.3.2 Semi-infinite plate thickness}

The coefficients of weight function $M$ at the ellipse vertex position in the plate width direction [point $\mathrm{C}(\varphi=$ $0)$ in Fig. 14] and free surface side [point B $(\varphi=-\pi / 2)$ in Fig. 14] and at the same position on the opposite side [point $\mathrm{A}(\varphi=\pi / 2)$ in Fig. 14] are given as polynomial approximations, where $\eta=d / a$ and $\alpha=a / c$.

A.3.2.1 Point $\mathbf{A}(\varphi=-\pi / 2)$ in Fig. 14

$$
\begin{aligned}
& M=E_{1}+E_{2} \alpha+E_{3} \eta+E_{4} \alpha^{2}+E_{5} \eta^{2}+E_{6} \alpha \eta^{2}+E_{7} \alpha^{2} \eta^{2} \\
& E_{1}=1.8626, E_{2}=-1.0554, E_{3}=-3.5249, E_{4}=0.22694, \\
& E_{5}=1.5989, E_{6}=2.6770, E_{7}=-1.1220
\end{aligned}
$$


A.3.2.2 Point $B(\varphi=\pi / 2)$ in Fig. 14

$$
\begin{gathered}
M=F_{1}+F_{2} \alpha^{-1}+F_{3} \eta+F_{4} \alpha^{-2}+F_{5} \eta^{2}+F_{6} \alpha^{-1} \eta+E_{7} \alpha^{-2} \eta \\
F_{1}=-0.37155, F_{2}=0.39094, F_{3}=0.095016, F_{4}=-0.055900, \\
F_{5}=0.076620, F_{6}=-0.26694, F_{7}=0.032455
\end{gathered}
$$

A.3.2.3 Point $C(\varphi=0)$ in Fig. 14

$$
\begin{gathered}
M=G_{1}+G_{2} \alpha^{-1}+G_{3} \eta+G_{4} \alpha^{-2}+G_{5} \eta^{2}+G_{6} \alpha^{-1} \eta \\
G_{1}=0.07905, G_{2}=-0.022090, G_{3}=-0.10460, G_{4}=-0.067760, \\
G_{5}=0.076620, G_{6}=-0.028640, G_{7}=0.050680
\end{gathered}
$$

\section{Acknowledgement}

This paper owes much to the thoughtful and helpful support for finite element analysis by Professor Xin

Wang, Department of Mechanical and Aerospace Engineering, Carleton University, Canada.

\section{References}

[1] Newman Jr JC, Raju IS. An empirical stress intensity factor equation for the surface crack. Eng Fract Mech 1981; 15:185-92.

[2] Newman Jr JC, Raju IS. Stress-intensity factor equations for cracks in three-dimensional finite bodies, Fracture Mechanics, 14th Symposium Vol. I, ASTM STP 791 1983; I238-65.

[3] Bueckner HF. A novel principle for the computation of stress intensity factors. Z Angew Math Mech 1970; 50:129-46.

[4] Rice J. Some remarks on elastic crack tip field. Int J Solids Struct 1972; 8:751-8.

[5] Wu XR, Carlsson AJ. Weight functions and stress intensity factor solutions. Oxford: Pergamon Press; 1991. 
[6] Fett T, Munz D. Stress intensity factors and weight functions. Southampton: Computational Mechanics Publications; 1997.

[7] Glinka G, Shen G. Universal features of weight functions for cracks in mode I. Eng Fract Mech 1991; 40:1135-46.

[8] Tada H, Paris PC, Irwin GR. The stress analysis of cracks handbook. 3rd Ed. New York: ASME Press; 2007.

[9] Rice J. Weight function theory for three-dimensional elastic crack analysis. In: Fracture mechanics perspectives and directions (20th symposium), ASTM STP 1020; 1989. 29-57.

[10] Wang X, Glinka G. Determination of approximate point load weight functions for embedded elliptical cracks. Int J Fatigue 2009; 31:1816-27.

[11] Gotoh K, Otaguro T. Improved point load weight function for stress intensity factor of a surface and an embedded crack. J Japan Society Naval Architects and Ocean Engineers 2013; 17:65-73. (in Japanese)

[12] Shratori M, Miyoshi T. Analysis of Stress Intensity Factors for Surface Cracks Subjected to Arbitrarily Distributed Stresses Computational Mechanics '86 pp 1027-1032

[13] Nagai M, Miura N, Shiratori M. Stress intensity factor solution for a surface crack with high aspect ratio subjected to an arbitrary stress distribution using the influence function method: International Journal of Pressure Vessels and Piping, Volume 131, July 2015, Pages 2-9

[14] https://support.office.com/en-us/article/define-and-solve-a-problem-by-using-solver-5d1a388f-079d43ac-a7eb-f63e45925040 (accessed on April 5, 2019).

[15] https://www.mscsoftware.com/product/marc [accessed on October 15, 2019].

[16] https://www.3ds.com/products-services/simulia/products/abaqus/abaqusstandard/ [accessed on October $15,2019]$. 